\title{
Existence of Mild Solutions to Nonlocal Fractional Cauchy Problems via Compactness
}

\author{
Rodrigo Ponce \\ Instituto de Matemática y Física, Universidad de Talca, Casilla 747, Talca, Chile \\ Correspondence should be addressed to Rodrigo Ponce; rponce@inst-mat.utalca.cl
}

Received 7 July 2016; Accepted 22 August 2016

Academic Editor: Khalil Ezzinbi

Copyright ( 2016 Rodrigo Ponce. This is an open access article distributed under the Creative Commons Attribution License, which permits unrestricted use, distribution, and reproduction in any medium, provided the original work is properly cited.

We obtain characterizations of compactness for resolvent families of operators and as applications we study the existence of mild solutions to nonlocal Cauchy problems for fractional derivatives in Banach spaces. We discuss here simultaneously the Caputo and Riemann-Liouville fractional derivatives in the cases $0<\alpha<1$ and $1<\alpha<2$.

\section{Introduction}

The nonlocal initial conditions were introduced to extend the classical theory of initial value problems. Nonlocal conditions describe more appropriately some natural phenomena because they consider additional information in the initial conditions.

The existence of mild solutions to semilinear Cauchy problems with nonlocal conditions has been studied by several authors in the last two decades. See, for instance, [1-4] and the references cited therein.

On the other hand, many authors have studied recently the existence of mild solutions to abstract fractional differential equations with nonlocal conditions by using the theory of resolvent families of operators as well as some fixed point results. See [5-18] and the references therein for more details.

Let $A$ be a closed and linear operator defined on a Banach space $X, u_{0}, u_{1} \in X$, and $T>0$ and suppose that $f, p$, and $q$ are suitable continuous functions. In what follows, we will denote by $D_{t}^{\alpha}$ and $D^{\alpha}$ the Caputo and Riemann-Liouville fractional derivatives, respectively. Now, for $t \in[0, T]$, we consider the following nonlinear fractional differential equations with nonlocal conditions

$$
\begin{aligned}
D_{t}^{\alpha} u(t) & =A u(t)+f(t, u(t)), \\
u(0) & =p(u)+u_{0},
\end{aligned}
$$

$$
\begin{aligned}
D^{\alpha} u(t) & =A u(t)+f(t, u(t)), \\
\left(g_{1-\alpha} * u\right)(0) & =p(u)+u_{0},
\end{aligned}
$$

in case $0<\alpha<1$; and

$$
\begin{aligned}
D_{t}^{\alpha} u(t) & =A u(t)+f(t, u(t)), \\
u(0) & =p(u)+u_{0}, \\
u^{\prime}(0) & =q(u)+u_{1}, \\
D^{\alpha} u(t) & =A u(t)+f(t, u(t)), \\
\left(g_{2-\alpha} * u\right)(0) & =p(u)+u_{0}, \\
\left(g_{2-\alpha} * u\right)^{\prime}(0) & =q(u)+u_{1},
\end{aligned}
$$

in case $1<\alpha<2$.

By using the Laplace transform, it is easy to see that the mild solutions to problems (1)-(4) are, respectively, given by

$$
\begin{aligned}
u(t)= & S_{\alpha, 1}(t)\left(u_{0}+p(u)\right) \\
& +\int_{0}^{t} S_{\alpha, \alpha}(t-s) f(s, u(s)) d s, \\
u(t)= & S_{\alpha, \alpha}(t)\left(u_{0}+(u)\right) \\
& +\int_{0}^{t} S_{\alpha, \alpha}(t-s) f(s, u(s)) d s,
\end{aligned}
$$


in case $0<\alpha<1$; and

$$
\begin{aligned}
u(t)= & S_{\alpha, 1}(t)\left(u_{0}+p(u)\right)+S_{\alpha, 2}(t)\left(u_{1}+q(u)\right) \\
& +\int_{0}^{t} S_{\alpha, \alpha}(t-s) f(s, u(s)) d s, \\
u(t)= & S_{\alpha, \alpha-1}(t)\left(u_{0}+p(u)\right)+S_{\alpha, \alpha}(t)\left(u_{1}+q(u)\right) \\
& +\int_{0}^{t} S_{\alpha, \alpha}(t-s) f(s, u(s)) d s,
\end{aligned}
$$

in case $1<\alpha<2$. Here, for $\alpha, \beta>0,\left\{S_{\alpha, \beta}(t)\right\}_{t \geq 0}$ is the resolvent family generated by $A$ (see definition below, Section 2).

The existence of mild solutions to problems (1)-(4) has been studied by many authors in the last years. For example, in case $0<\alpha<1$, we refer the reader to $[8,9,17,18]$ (for the Caputo fractional derivative) and to [10] (for the RiemannLiouville fractional derivative), that is, problems (1) and (2), respectively. On the other hand, in case $1<\alpha<2$, the existence of mild solutions to the Caputo fractional Cauchy problems with nonlocal conditions (3) has been considered in $[12,19]$ and the references therein, and, to the best of our knowledge, nonlocal Riemann-Liouville fractional Cauchy problem (4) has not been addressed in the existing literature.

A common assumption in many of the above-mentioned papers to obtain the existence of mild solutions to problems (1)-(4) is that $A$ generates a compact analytic semigroup $\{T(t)\}_{t \geq 0}$, or $A$ generates a compact fractional resolvent family $\left\{S_{\alpha, 1}(t)\right\}_{t \geq 0}$ (see the definition below) because the compactness of $\{T(t)\}_{t \geq 0}$ (or $\left\{S_{\alpha, 1}(t)\right\}_{t \geq 0}$ ) allows applying, for example, the Krasnoselskii fixed point theorem.

According to the variation of constants formulas (5)-(7), we observe that if we have compactness criteria of $S_{\alpha, \beta}(t)$ (for suitable $\alpha$ and $\beta$ ), we will be able to apply some fixed point techniques to obtain the existence of mild solutions to problems (1)-(4). For example, to prove the existence of mild solutions to problem (3), the authors in [12, Theorem 1.2] assume that the operators $S_{\alpha, 1}(t), S_{\alpha, 2}(t)$, and $S_{\alpha, \alpha}(t)$ generated by $A$ are compact for all $t>0$. However, there are not completely clear conditions on $A$ implying the compactness of $S_{\alpha, 1}(t), S_{\alpha, 2}(t)$, and $S_{\alpha, \alpha}(t)$ for all $t>0$, because there are no compactness criteria for $S_{\alpha, \beta}(t)$, when $\alpha, \beta>0$. Therefore, we notice that the compactness of $S_{\alpha, \beta}(t)$ gives a powerful tool to obtain existence of mild solutions to problems (1)-(4).

The compactness of $S_{\alpha, \beta}(t)$ is well known in some special cases. For example, if $\alpha=\beta=1$, then $S_{1,1}(t)$ is compact for all $t>0$ if and only if $S_{1,1}(t)$ is norm continuos and $(\lambda-A)^{-1}$ is compact for all $\lambda \in \rho(A)$, because $\left\{S_{1,1}(t)\right\}_{t \geq 0}$ corresponds to a $C_{0}$-semigroup. See [20, Theorem 3.3, Chapter 2]. If $\alpha=\beta=2$, then $S_{2,2}(t)$ is compact for all $t>0$ if and only if $\left(\lambda^{2}-A\right)^{-1}$ is compact $\lambda \in \rho(A)$, because $\left\{S_{2,2}(t)\right\}_{t \geq 0}$ is the sine family generated by $A$; see [21]. In case $0<\alpha<1$, the compactness of $S_{\alpha, 1}(t)$ has been studied by using subordination methods; that is, the operator $A$ is supposed to be a generator of a compact semigroup; see [22]. On the other hand, if $A$ is an almost sectorial operator and the resolvent $\left(\lambda^{\alpha}-A\right)^{-1}$ is compact for all $\lambda \in \rho(A)$, then $S_{\alpha, 1}(t)$ is compact for all $t>0$ (see [23]), and, very recently, it was proved that if $S_{\alpha, 1}(t)$ is norm continuous, then $S_{\alpha, 1}(t)$ is compact for all $t>0$ if and only if $\left(\lambda^{\alpha}-A\right)^{-1}$ is compact for all $\lambda \in \rho(A)$. See [24, 25]. Finally, in case $1<\alpha<2$, the characterization of compactness asserts that $S_{\alpha, \alpha}(t)$ is compact for all $t>0$ if and only if $\left(\lambda^{\alpha}-A\right)^{-1}$ is compact for all $\lambda \in \rho(A)$; see [25, Theorem 3.5].

In this paper, we study the existence of mild solution to nonlocal fractional Cauchy problems (1)-(4). Our approach relies on the compactness of resolvent family $\left\{S_{\alpha, \beta}(t)\right\}_{t \geq 0}$ for suitable $\alpha, \beta>0$, as well as some fixed point techniques. We remark that we study simultaneously the nonlocal fractional Cauchy problem for the Caputo and Riemann-Liouville fractional derivatives.

The paper is organized as follows. Section 2 gives the preliminaries. Section 3 is devoted to the norm continuity and compactness of $S_{\alpha, \beta}(t)$ for $t>0$. Here, we give characterizations of the compactness of $S_{\alpha, \beta}(t)$ for $t>0$ for suitable $\alpha, \beta>$ 0 . In Section 4 we study nonlocal fractional Cauchy problems for the Caputo fractional derivative. We give some results on the existence of mild solutions to problems (1) and (3). Section 5 treats nonlocal fractional Cauchy problems for the Riemann-Liouville fractional derivative. Here, we study the existence of mild solutions to problems (2) and (4). Finally, Section 6 is devoted to some applications.

\section{Preliminaries}

Let $(X,\|\cdot\|)$ be a Banach space. We denote by $\mathscr{B}(X)$ the space of all bounded linear operators from $X$ into $X$. If $A$ is a closed linear operator on $X$, we denote by $\rho(A)$ the resolvent set of $A$ and $R(\lambda, A)=(\lambda-A)^{-1}$ the resolvent operator of $A$ defined for all $\lambda \in \rho(A)$.

We recall that a strongly continuous family $\{S(t)\}_{t \geq 0} \subset$ $\mathscr{B}(X)$ is said to be of type $(M, \omega)$ or is exponentially bounded, if there exist two constants $M>0$ and $w \in \mathbb{R}$ such that $\|S(t)\| \leq M e^{w t}$ for all $t>0$.

Now, we review some results on fractional calculus. For $\mu>0$, define

$$
g_{\mu}(t)= \begin{cases}\frac{t^{\mu-1}}{\Gamma(\mu)}, & t>0 \\ 0, & t \leq 0\end{cases}
$$

where $\Gamma(\cdot)$ is the Gamma function. We define $g_{0} \equiv \delta_{0}$, the Dirac delta. For $\mu>0, n=\lceil\mu\rceil$ denotes the smallest integer $n$ greater than or equal to $\mu$. As usual, the finite convolution of $f$ and $g$ is defined by $(f * g)(t)=\int_{0}^{t} f(t-s) g(s) d s$.

Definition 1. Let $\alpha>0$. The $\alpha$-order Riemann-Liouville fractional integral of $u$ is defined by

$$
J^{\alpha} u(t):=\int_{0}^{t} g_{\alpha}(t-s) u(s) d s, \quad t \geq 0
$$

Also, we define $J^{0} u(t)=u(t)$. Because of the convolution properties, the integral operators $\left\{J^{\alpha}\right\}_{\alpha \geq 0}$ satisfy the semigroup law: $J^{\alpha} J^{\beta}=J^{\alpha+\beta}$ for all $\alpha, \beta \geq 0$. 
Definition 2. Let $\alpha>0$. The $\alpha$-order Caputo fractional derivative is defined as

$$
D_{t}^{\alpha} u(t):=\int_{0}^{t} g_{n-\alpha}(t-s) u^{(n)}(s) d s
$$

where $n=\lceil\alpha\rceil$.

Definition 3. Let $\alpha>0$. The $\alpha$-order Riemann-Liouville fractional derivative of $u$ is defined as

$$
D^{\alpha} u(t):=\frac{d^{n}}{d t^{n}} \int_{0}^{t} g_{n-\alpha}(t-s) u(s) d s,
$$

where $n=\lceil\alpha\rceil$.

We notice that if $\alpha=m \in \mathbb{N}$, then $D_{t}^{m}=D^{m}=d^{m} / d t^{m}$.

Throughout this paper we use the notation of $D_{t}^{\alpha}$ and $D^{\alpha}$ to the $\alpha$-fractional derivative of Caputo and RiemannLiouville, respectively.

Example 4. If $\alpha, \beta>0$, then

(i) $J^{\alpha} t^{\beta}=(\Gamma(\beta+1) / \Gamma(\alpha+\beta+1)) t^{\alpha+\beta}$,

(ii) $D^{\alpha} t^{\beta}=(\Gamma(\beta+1) / \Gamma(\alpha+\beta+1)) t^{\beta-\alpha}=D_{t}^{\alpha} t^{\beta}$.

(iii) $D_{t}^{\alpha} e^{\rho t}=\rho^{2} t^{2-\alpha} e_{1,3-\alpha}(\rho t)$.

We observe that the Riemann-Liouville derivative operator $D^{\alpha}$ is a left inverse operator of $J^{\alpha}$ but not a right inverse, that is,

$$
\begin{aligned}
D^{\alpha} J^{\alpha} u(t) & =u(t), \\
\left(J^{\alpha} D^{\alpha}\right) u(t)= & u(t) \\
& -\sum_{k=0}^{n-1}\left(g_{n-\alpha} * u\right)^{(k)}(0) g_{\alpha+1+k-n}(t),
\end{aligned}
$$

$n=\lceil\alpha\rceil$. On the other hand, the Caputo derivative operator $D_{t}^{\alpha}$ satisfies

$$
\begin{aligned}
D_{t}^{\alpha} J^{\alpha} u(t) & =u(t), \\
\left(J^{\alpha} D_{t}^{\alpha}\right) u(t) & =u(t)-\sum_{k=0}^{n-1} u^{(k)}(0) g_{k+1}(t) .
\end{aligned}
$$

If we denote by $\widehat{f}$ (or $\mathscr{L}(f))$ the Laplace transform of $f$, we have the following properties for the fractional derivatives:

$$
\begin{aligned}
& \widehat{D^{\alpha} u}(\lambda)=\lambda^{\alpha} \widehat{u}(\lambda)-\sum_{k=0}^{n-1}\left(g_{n-\alpha} * u\right)^{(k)}(0) \lambda^{n-1-k}, \\
& \widehat{D_{t}^{\alpha} u}(\lambda)=\lambda^{\alpha} \widehat{u}(\lambda)-\sum_{k=0}^{n-1} u^{(k)}(0) \lambda^{\alpha-1-k},
\end{aligned}
$$

where $n=\lceil\alpha\rceil$ and $\lambda \in \mathbb{C}$. For $\alpha, \beta>0$ and $z \in \mathbb{C}$, the generalized Mittag-Leffler function is defined by

$$
e_{\alpha, \beta}(z):=\sum_{k=0}^{\infty} \frac{z^{k}}{\Gamma(\alpha k+\beta)} .
$$

The Laplace transform $\mathscr{L}$ of the Mittag-Leffler function satisfies

$$
\mathscr{L}\left(t^{\beta-1} e_{\alpha, \beta}\left(\rho t^{\alpha}\right)\right)(\lambda)=\frac{\lambda^{\alpha-\beta}}{\lambda^{\alpha}-\rho},
$$

$$
\rho \in \mathbb{C}, \operatorname{Re} \lambda>|\rho|^{1 / \alpha} .
$$

Definition 5. Let $A$ be closed linear operator with domain $D(A)$, defined on a Banach space $X$, and $\alpha, \beta>0$. We say that $A$ is the generator of an $(\alpha, \beta)$-resolvent family, if there exist $\omega \geq 0$ and a strongly continuous function $S_{\alpha, \beta}$ : $[0, \infty) \rightarrow \mathscr{B}(X)$ such that $\left\{S_{\alpha, \beta}(t)\right\}$ is exponentially bounded, $\left\{\lambda^{\alpha}: \operatorname{Re} \lambda>\omega\right\} \subset \rho(A)$, and, for all $x \in X$,

$$
\lambda^{\alpha-\beta}\left(\lambda^{\alpha}-A\right)^{-1} x=\int_{0}^{\infty} e^{-\lambda t} S_{\alpha, \beta}(t) x d t, \quad \operatorname{Re} \lambda>\omega .
$$

In this case, $\left\{S_{\alpha, \beta}(t)\right\}_{t \geq 0}$ is called the $(\alpha, \beta)$-resolvent family generated by $A$.

We notice that Definition 5 corresponds to the concept of $(a, k)$-regularized families introduced in [26]. In fact, if $a=$ $g_{\alpha}$ and $b=g_{\beta}$, then the function $t \mapsto S_{\alpha, \beta}(t)$ is a $\left(g_{\alpha}, g_{\beta}\right)$ regularized family. Moreover, the function $S_{\alpha, \beta}(t)$ satisfies the following functional equation (see $[27,28]$ ):

$$
\begin{aligned}
& S_{\alpha, \beta}(s)\left(g_{\alpha} * S_{\alpha, \beta}\right)(t)-\left(g_{\alpha} * S_{\alpha, \beta}\right)(s) S_{\alpha, \beta}(s) \\
& =g_{\beta}(s)\left(g_{\alpha} * S_{\alpha, \beta}\right)(t)-g_{\beta}(t)\left(g_{\alpha} * S_{\alpha, \beta}\right)(s),
\end{aligned}
$$

for all $t, s \geq 0$. On the other hand, if an operator $A$ with domain $D(A)$ is the infinitesimal generator of the $(\alpha, \beta)$ resolvent family $S_{\alpha, \beta}(t)$, then for all $x \in D(A)$ we have

$$
A x=\lim _{t \rightarrow 0^{+}} \frac{S_{\alpha, \beta}(t) x-g_{\beta}(t) x}{g_{\alpha+\beta}(t)} .
$$

For example, the case $S_{1,1}(t)$ corresponds to a $C_{0}$-semigroup and $S_{2,1}(t)$ is a cosine family, whereas $S_{2,2}(t)$ is a sine family. Finally, if $\beta=1$, then $S_{\alpha, 1}(t)$ is the $\alpha$-resolvent family (also called the $\alpha$-times resolvent family) for fractional differential equations. We notice that, in the scalar case, that is, when $A=$ $\rho I$, where $\rho \in \mathbb{C}$ and $I$ denotes the identity operator, then by the uniqueness of the Laplace transform $S_{\alpha, \beta}(t)$ corresponds to the function $t^{\beta-1} e_{\alpha, \beta}\left(\rho t^{\alpha}\right)$. Finally, let $0<\alpha<1$ and $\beta \geq \alpha$. Define $\left\{S_{\alpha, \beta}(t)\right\}_{t \geq 0}$ by

$$
S_{\alpha, \beta}(t) f(s):=\int_{0}^{s} f(s-r) \varphi_{\alpha, \beta-\alpha}(t, r) d r,
$$

where $s \in \mathbb{R}_{+}, f \in L^{1}\left(\mathbb{R}_{+}\right)$, and the function $\varphi_{a, b}(t, r)$ is defined by

$$
\varphi_{a, b}(t, r):=t^{b-1} W_{-a, b}\left(-r t^{-a}\right), \quad a>0, \quad b \geq 0,
$$

where $W_{-a, b}(z):=\sum_{n=0}^{\infty}\left(z^{n} / n ! \Gamma(-a n+b)\right)(z \in \mathbb{C})$ denotes the Wright function. Then, $\left\{S_{\alpha, \beta}(t)\right\}_{t \geq 0}$ is an $(\alpha, \beta)$-resolvent family on the Banach space $X=L^{1}\left(\mathbb{R}_{+}\right)$generated by $A=$ $-d / d t$. See [29, Example 11].

The proof of the next result follows as in $[26,27]$. 
Proposition 6. Let $\alpha, \beta>0$ and let $\left\{S_{\alpha, \beta}(t)\right\}_{t \geq 0} \subset \mathscr{B}(X)$ be an $(\alpha, \beta)$-resolvent family generated by $A$. Then the following holds:

(1) $S_{\alpha, \beta}(t) x \in D(A)$ and $S_{\alpha, \beta}(t) A x=A S_{\alpha, \beta}(t) x$ for all $x \in D(A)$ and $t \geq 0$.

(2) If $x \in D(A)$ and $t \geq 0$, then

$$
S_{\alpha, \beta}(t) x=g_{\beta}(t) x+\int_{0}^{t} g_{\alpha}(t-s) A S_{\alpha, \beta}(s) x d s .
$$

(3) If $x \in X$ and $t \geq 0$, then $\int_{0}^{t} g_{\alpha}(t-s) S_{\alpha, \beta}(s) x d s \in D(A)$, and

$$
S_{\alpha, \beta}(t) x=g_{\beta}(t) x+A \int_{0}^{t} g_{\alpha}(t-s) S_{\alpha, \beta}(s) x d s .
$$

In particular, $S_{\alpha, \beta}(0)=g_{\beta}(0) I$.

Finally, we recall the following results.

Theorem 7 (Mazur theorem). If $K$ is a compact subset of a Banach space $X$, then its convex closure $\overline{\operatorname{conv}(K)}$ is compact.

Theorem 8 (Krasnoselskii theorem). Let $C$ be a closed convex and nonempty subset of a Banach space $X$. Let $Q_{1}$ and $Q_{2}$ be two operators such that

(i) if $u, v \in C$, then $Q_{1} u+Q_{2} v \in C$,

(ii) $Q_{1}$ is a mapping contraction,

(iii) $Q_{2}$ is compact and continuous.

Then, there exists $z \in C$ such that $z=Q_{1} z+Q_{2} z$.

Theorem 9 (Schauder's fixed point theorem). Let $C$ be a nonempty, closed, bounded, and convex subset of a Banach space $X$. Suppose that $\Gamma: C \rightarrow C$ is a compact operator. Then $\Gamma$ has at least a fixed point in $C$.

Theorem 10 (Leray-Schauder alternative theorem). Let $C$ be a convex subset of a Banach space $X$. Suppose that $0 \in C$. If $\Gamma: C \rightarrow C$ is a completely continuous map, then either $\Gamma$ has a fixed point or the set $\{x \in C: x=\lambda \Gamma(x), 0<\lambda<1\}$ is unbounded.

\section{Continuity and Compactness of $S_{\alpha, \beta}(t)$}

In this section we study, for all $t>0$, the norm continuity (continuity in $\mathscr{B}(X)$ ) and the compactness of $S_{\alpha, \beta}(t)$ for given $\alpha, \beta>0$.

Proposition 11. Let $\alpha>0$ and $1<\beta \leq 2$. Suppose that $\left\{S_{\alpha, \beta}(t)\right\}_{t \geq 0}$ is the $(\alpha, \beta)$-resolvent family of type $(M, \omega)$ generated by $A$. Then the function $t \mapsto S_{\alpha, \beta}(t)$ is continuous in $\mathscr{B}(X)$ for all $t>0$.
Proof. Let $1<\beta<2$. Observe that, for all $\operatorname{Re} \lambda>0$,

$$
\begin{aligned}
\mathscr{L}\left(S_{\alpha, \beta}\right)(\lambda) & =\lambda^{\alpha-\beta}\left(\lambda^{\alpha}-A\right)^{-1} \\
& =\frac{1}{\lambda^{\beta-1}} \lambda^{\alpha-1}\left(\lambda^{\alpha}-A\right)^{-1} \\
& =\mathscr{L}\left(g_{\beta-1} * S_{\alpha, 1}\right)(\lambda) .
\end{aligned}
$$

We conclude by the uniqueness of the Laplace transform that $S_{\alpha, \beta}(t)=\left(g_{\beta-1} * S_{\alpha, 1}\right)(t)$, for all $t>0$. Take $0<t_{0}<t_{1}$. Then

$$
\begin{aligned}
S_{\alpha, \beta} & \left(t_{1}\right)-S_{\alpha, \beta}\left(t_{0}\right) \\
= & \left(g_{\beta-1} * S_{\alpha, 1}\right)\left(t_{1}\right)-\left(g_{\beta-1} * S_{\alpha, 1}\right)\left(t_{0}\right) \\
= & \int_{t_{0}}^{t_{1}} g_{\beta-1}\left(t_{1}-r\right) S_{\alpha, 1}(r) d r \\
& \quad+\int_{0}^{t_{0}}\left[g_{\beta-1}\left(t_{1}-r\right)-g_{\beta-1}\left(t_{0}-r\right)\right] S_{\alpha, 1}(r) d r \\
= & I_{1}+I_{2} .
\end{aligned}
$$

Since $\beta>1$, we have $g_{\beta}(0)=0$ and we obtain

$$
\begin{aligned}
\left\|I_{1}\right\| & \leq \int_{t_{0}}^{t_{1}} g_{\beta-1}\left(t_{1}-r\right)\left\|S_{\alpha, 1}(r)\right\| d r \\
& \leq M \int_{t_{0}}^{t_{1}} g_{\beta-1}\left(t_{1}-r\right) e^{\omega r} d r=M e^{\omega t_{1}} g_{\beta}\left(t_{1}-t_{0}\right),
\end{aligned}
$$

and therefore $\left\|I_{1}\right\| \rightarrow 0$ as $t_{1} \rightarrow t_{0}$.

On the other hand,

$$
\begin{aligned}
\left\|I_{2}\right\| & \leq \int_{0}^{t_{0}}\left|g_{\beta-1}\left(t_{1}-r\right)-g_{\beta-1}\left(t_{0}-r\right)\right|\left\|S_{\alpha, 1}(r)\right\| d r \\
& \leq M e^{\omega t_{1}} \int_{0}^{t_{0}}\left|g_{\beta-1}\left(t_{1}-r\right)-g_{\beta-1}\left(t_{0}-r\right)\right| d r \\
& =M e^{\omega t_{1}} \int_{0}^{t_{0}}\left|g_{\beta-1}\left(t_{1}-t_{0}+r\right)-g_{\beta-1}(r)\right| d r .
\end{aligned}
$$

Since $1<\beta<2$ we obtain that the function $r \mapsto g_{\beta-1}(r)$ is decreasing in $[0, \infty)$ and therefore $g_{\beta-1}(r)-g_{\beta-1}\left(t_{1}-t_{0}+r\right)>$ 0 , for all $r>0$, obtaining

$$
\begin{aligned}
\left\|I_{2}\right\| & \leq M e^{\omega t_{1}} \int_{0}^{t_{0}}\left[g_{\beta-1}(r)-g_{\beta-1}\left(t_{1}-t_{0}+r\right)\right] d r \\
& =M e^{\omega t_{1}}\left[g_{\beta}\left(t_{0}\right)-g_{\beta}\left(t_{1}\right)+g_{\beta}\left(t_{1}-t_{0}\right)\right] .
\end{aligned}
$$

Therefore, $\left\|I_{2}\right\| \rightarrow 0$ as $t_{1} \rightarrow t_{0}$. We conclude that $S_{\alpha, \beta}(t)$ is norm continuous, for $1<\beta<2$.

On the other hand, if $\beta=2$, then, by the uniqueness of the Laplace transform, we obtain that

$$
S_{\alpha, 2}(t) x=\left(g_{1} * S_{\alpha, 1}\right)(t) x=\int_{0}^{t} S_{\alpha, 1}(r) x d r
$$


for all $x \in X$. Take $0<t_{0}<t_{1}$. Then

$$
\begin{aligned}
& \left\|S_{\alpha, 2}\left(t_{1}\right) x-S_{\alpha, 2}\left(t_{0}\right) x\right\| \leq \int_{t_{0}}^{t_{1}}\left\|S_{\alpha, 1}(r) x\right\| d r \\
& \quad \leq M e^{\omega t_{1}}\|x\|\left(t_{1}-t_{0}\right)
\end{aligned}
$$

for all $x \in X$. Therefore $\left\|S_{\alpha, 2}\left(t_{1}\right)-S_{\alpha, 2}\left(t_{0}\right)\right\| \rightarrow 0$ as $t_{1} \rightarrow$ $t_{0}$.

Lemma 12. Suppose that $A$ generates an $(\alpha, \beta)$-resolvent family $\left\{S_{\alpha, \beta}(t)\right\}_{t \geq 0}$ of type $(M, \omega)$. If $\gamma>0$, then A generates an $(\alpha, \beta+\gamma)$-resolvent family of type $\left(M / \omega^{\gamma}, \omega\right)$.

Proof. By hypothesis we get, for all $t \geq 0$,

$$
\begin{aligned}
\left\|\left(g_{\gamma} * S_{\alpha, \beta}\right)(t)\right\| & \leq M \int_{0}^{t} g_{\gamma}(t-s) e^{\omega s} d s \\
& \leq M e^{\omega t} \int_{0}^{t} g_{\gamma}(s) e^{-\omega s} d s \\
& \leq M e^{\omega t} \int_{0}^{\infty} g_{\gamma}(s) e^{-\omega s} d s=\frac{M e^{\omega t}}{\omega^{\gamma}} .
\end{aligned}
$$

Therefore $\left(g_{\gamma} * S_{\alpha, \beta}\right)(t)$ is Laplace transformable and, for all $\lambda>\omega$, we have

$$
\begin{aligned}
\mathscr{L}\left(g_{\gamma} * S_{\alpha, \beta}\right)(\lambda) & =\frac{1}{\lambda^{\gamma}} \lambda^{\alpha-\beta}\left(\lambda^{\alpha}-A\right)^{-1} \\
& =\lambda^{\alpha-(\beta+\gamma)}\left(\lambda^{\alpha}-A\right)^{-1} \\
& =\mathscr{L}\left(S_{\alpha, \beta+\gamma}\right)(\lambda) .
\end{aligned}
$$

We conclude that $A$ generates an $(\alpha, \beta+\gamma)$-resolvent family of type $\left(M / \omega^{\gamma}, \omega\right)$.

Definition 13. We say that the resolvent family $\left\{S_{\alpha, \beta}(t)\right\}_{t \geq 0} \subset$ $\mathscr{B}(X)$ is compact if, for every $t>0$, the operator $S_{\alpha, \beta}(t)$ is a compact operator.

In what follows, we will assume that $\left\{S_{\alpha, \beta}(t)\right\}_{t \geq 0}$ is strongly continuous for all $\alpha, \beta>0$.

Theorem 14. Let $\alpha>0,1<\beta \leq 2$, and $\left\{S_{\alpha, \beta}(t)\right\}_{t \geq 0}$ be an $(\alpha, \beta)$-resolvent family of type $(M, \omega)$ generated by $A$. Then the following assertions are equivalent:

(i) $S_{\alpha, \beta}(t)$ is a compact operator for all $t>0$.

(ii) $(\mu-A)^{-1}$ is a compact operator for all $\mu>\omega^{1 / \alpha}$.

Proof. (i) $\Rightarrow$ (ii) Suppose that the resolvent family $\left\{S_{\alpha, \beta}(t)\right\}_{t>0}$ is compact. Let $\lambda>\omega$ be fixed. Then we have

$$
\lambda^{\alpha-\beta}\left(\lambda^{\alpha}-A\right)^{-1}=\int_{0}^{\infty} e^{-\lambda t} S_{\alpha, \beta}(t) d t
$$

where the integral in the right-hand side exists in the Bochner sense. Because $\left\{S_{\alpha, \beta}(t)\right\}_{t>0}$ is continuous in the uniform operator topology (by Proposition 11), we conclude that $\left(\lambda^{\alpha}-\right.$ $A)^{-1}$ is a compact operator by [30, Corollary 2.3]. (ii) $\Rightarrow$ (i) Let $t>0$ be fixed. Assume that $1<\beta<2$. Since $\beta>1$, it follows that $g_{\beta-1} \in L_{\text {loc }}^{1}[0, \infty)$ and therefore, by [31, Proposition 2.1], we obtain

$$
\begin{gathered}
\lim _{N \rightarrow \infty} \frac{1}{2 \pi i} \int_{\omega-i N}^{\omega+i N} e^{\lambda t}\left(\bar{g}_{\beta-1} * S_{\alpha, 1}\right)(\lambda) d \lambda \\
=\left(g_{\beta-1} * S_{\alpha, 1}\right)(t)=S_{\alpha, \beta}(t),
\end{gathered}
$$

in $\mathscr{B}(X)$. Therefore,

$$
\frac{1}{2 \pi i} \int_{\Gamma} e^{\lambda t} \lambda^{\alpha-\beta}\left(\lambda^{\alpha}-A\right)^{-1} d \lambda=S_{\alpha, \beta}(t), \quad t>0
$$

where $\Gamma$ is the path consisting of the vertical line $\{\omega+i s: s \in$ $\mathbb{R}\}$. By hypothesis and [30, Corollary 2.3], we conclude that $S_{\alpha, \beta}(t)$ is compact for all $\alpha>0$ and $1<\beta<2$. Now, we take $\beta=2$. Observe that in $\mathscr{B}(X)$ we have

$$
\begin{aligned}
& \lim _{N \rightarrow \infty} \frac{1}{2 \pi i} \int_{\omega-i N}^{\omega+i N} e^{\lambda t}\left(\widehat{g_{1} * S_{\alpha, 1}}\right)(\lambda) d \lambda=\left(g_{1} * S_{\alpha, 1}\right)(t) \\
& \quad=S_{\alpha, 2}(t)
\end{aligned}
$$

by [31, Proposition 2.1], and as in case $1<\beta<2$ we conclude that $S_{\alpha, 2}(t)$ is compact for all $t>0$.

By Theorem 14 we have the following corollary.

Corollary 15. Let $1<\alpha \leq 2$ and $\left\{S_{\alpha, \alpha}(t)\right\}_{t \geq 0}$ be an $(\alpha, \alpha)$ resolvent family of type $(M, \omega)$ generated by $A$. Then the following assertions are equivalent:

(i) $S_{\alpha, \alpha}(t)$ is a compact operator for all $t>0$.

(ii) $(\mu-A)^{-1}$ is a compact operator for all $\mu>\omega^{1 / \alpha}$.

Proposition 16. Let $1<\alpha<2$, and $\left\{S_{\alpha, 1}(t)\right\}_{t \geq 0}$ be the $(\alpha, 1)$ resolvent family of type $(M, \omega)$ generated by $A$. Suppose that $S_{\alpha, 1}(t)$ is continuous in the uniform operator topology for all $t>0$. Then the following assertions are equivalent:

(i) $S_{\alpha, 1}(t)$ is a compact operator for all $t>0$.

(ii) $(\mu-A)^{-1}$ is a compact operator for all $\mu>\omega^{1 / \alpha}$.

Proof. (i) $\Rightarrow$ (ii) Suppose that that the resolvent family $\left\{S_{\alpha, 1}(t)\right\}_{t>0}$ is compact. Let $\lambda>\omega$ be fixed. Then we have

$$
\lambda^{\alpha-1}\left(\lambda^{\alpha}-A\right)^{-1}=\int_{0}^{\infty} e^{-\lambda t} S_{\alpha, 1}(t) d t
$$


where the integral in the right-hand side exists in the Bochner sense, because $\left\{S_{\alpha, 1}(t)\right\}_{t>0}$ is continuous in the uniform operator topology, by hypothesis. Then, by [30, Corollary 2.3], we conclude that $\left(\lambda^{\alpha}-A\right)^{-1}$ is a compact operator.

(ii) $\Rightarrow$ (i) Let $t>0$ be fixed. Since $1<\alpha<2$, it follows that $g_{2-\alpha} \in L_{\text {loc }}^{1}[0, \infty)$ and therefore, by [31, Proposition 2.1], we obtain

$$
\begin{aligned}
& \lim _{N \rightarrow \infty} \frac{1}{2 \pi i} \int_{\omega-i N}^{\omega+i N} e^{\lambda t}\left(g_{2-\alpha} \widehat{* S_{\alpha, \alpha-1}}\right)(\lambda) d \lambda \\
& =\left(g_{2-\alpha} * S_{\alpha, \alpha-1}\right)(t)=S_{\alpha, 1}(t),
\end{aligned}
$$

in $\mathscr{B}(X)$. Therefore,

$$
\frac{1}{2 \pi i} \int_{\Gamma} e^{\lambda t} \lambda^{\alpha-1}\left(\lambda^{\alpha}-A\right)^{-1} d \lambda=S_{\alpha, 1}(t), \quad t>0,
$$

where $\Gamma$ is the path consisting of the vertical line $\{\omega+i s: s \in$ $\mathbb{R}\}$. By hypothesis and [30, Corollary 2.3], we conclude that $S_{\alpha, 1}(t)$ is compact.

Proposition 17. Let $3 / 2<\alpha<2$ and $\left\{S_{\alpha, \alpha-1}(t)\right\}_{t \geq 0}$ be the $(\alpha, \alpha-1)$-resolvent family of type $(M, \omega)$ generated by $A$. Suppose that $S_{\alpha, \alpha-1}(t)$ is continuous in the uniform operator topology for all $t>0$. Then the following assertions are equivalent:

(i) $S_{\alpha, \alpha-1}(t)$ is a compact operator for all $t>0$.

(ii) $(\mu-A)^{-1}$ is a compact operator for all $\mu>\omega^{1 / \alpha}$.

Proof. (i) $\Rightarrow$ (ii) It follows as in the proof of Proposition 16.

(ii) $\Rightarrow$ (i) Let $t>0$ be fixed. Since $\alpha>3 / 2$, it follows that $g_{\alpha-3 / 2} \in L_{\text {loc }}^{1}[0, \infty)$ and therefore, by [31, Proposition 2.1], we obtain

$$
\begin{gathered}
\lim _{N \rightarrow \infty} \frac{1}{2 \pi i} \int_{\omega-i N}^{\omega+i N} e^{\lambda t}\left(g_{\alpha-3 / 2} * S_{\alpha, 1 / 2}\right)(\lambda) d \lambda \\
=\left(g_{\alpha-3 / 2} * S_{\alpha, 1 / 2}\right)(t)=S_{\alpha, \alpha-1}(t),
\end{gathered}
$$

in $\mathscr{B}(X)$. Therefore,

$$
\frac{1}{2 \pi i} \int_{\Gamma} e^{\lambda t} \lambda^{\alpha-1}\left(\lambda^{\alpha}-A\right)^{-1} d \lambda=S_{\alpha, \alpha-1}(t),
$$

where $\Gamma$ is the path consisting of the vertical line $\{\omega+$ is $: s \in$ $\mathbb{R}\}$. By hypothesis and [30, Corollary 2.3], we conclude that $S_{\alpha, \alpha-1}(t)$ is compact.

The proof of the next result follows similarly to Proposition 16 , because for $1 / 2<\alpha<1$ we have

$$
\begin{gathered}
\lim _{N \rightarrow \infty} \frac{1}{2 \pi i} \int_{\omega-i N}^{\omega+i N} e^{\lambda t}\left(g_{\alpha-1 / 2} * S_{\alpha, 1 / 2}\right)(\lambda) d \lambda \\
\quad=\left(g_{\alpha-1 / 2} * S_{\alpha, 1 / 2}\right)(t)=S_{\alpha, \alpha}(t)
\end{gathered}
$$

in $\mathscr{B}(X)$ and $t>0$ by [31, Proposition 2.1].
Proposition 18. Let $1 / 2<\alpha<1$ and $\left\{S_{\alpha, \alpha}(t)\right\}_{t \geq 0}$ be the $(\alpha, \alpha)$ resolvent family of type $(M, \omega)$ generated by $A$. Suppose that $S_{\alpha, \alpha}(t)$ is continuous in the uniform operator topology for all $t>0$. Then, the following assertions are equivalent:

(i) $S_{\alpha, \alpha}(t)$ is a compact operator for all $t>0$.

(ii) $(\mu-A)^{-1}$ is a compact operator for all $\mu>\omega^{1 / \alpha}$.

Remark 19. Let $\varepsilon_{0}>0$ be fixed. If $\varepsilon_{0}<\alpha<1$, then by [31, Proposition 2.1] we have

$$
\begin{gathered}
\lim _{N \rightarrow \infty} \frac{1}{2 \pi i} \int_{\omega-i N}^{\omega+i N} e^{\lambda t}\left({\widehat{g} \alpha-\varepsilon_{0}}_{* S_{\alpha, \varepsilon_{0}}}\right)(\lambda) d \lambda \\
=\left(g_{\alpha-\varepsilon_{0}} * S_{\alpha, \varepsilon_{0}}\right)(t)=S_{\alpha, \alpha}(t)
\end{gathered}
$$

in $\mathscr{B}(X)$. Therefore, as is Proposition 18, if $\alpha>\varepsilon_{0}$, where $\varepsilon_{0}>0, A$ generates the $(\alpha, \alpha)$-resolvent family $\left\{S_{\alpha, \alpha}(t)\right\}_{t \geq 0}$ of type $(M, \omega)$, and $S_{\alpha, \alpha}(t)$ is norm continuous for all $t>0$, then $S_{\alpha, \alpha}(t)$ is a compact operator for all $t>0$ if and only if $\left(\lambda^{\alpha}-A\right)^{-1}$ is a compact operator for all $\lambda>\omega^{1 / \alpha}$. The same conclusion holds if $\varepsilon_{0}<\alpha<2$, where $\varepsilon_{0}>1$ is fixed and $\left\{S_{\alpha, \alpha-1}(t)\right\}_{t \geq 0}$ is the $(\alpha, \alpha-1)$-resolvent family of type $(M, \omega)$ generated by $A$, which is norm continuous for all $t>0$.

\section{Nonlocal Fractional Cauchy Problems: The Caputo Case}

In this section we consider the nonlocal problem for the Caputo fractional derivative

$$
\begin{aligned}
D_{t}^{\alpha} u(t) & =A u(t)+f(t, u(t)), \quad t \in I:=[0, T], \\
u(0)+p(u) & =u_{0}, \\
u^{\prime}(0)+q(u) & =u_{1},
\end{aligned}
$$

$u_{0}, u_{1} \in X, 1<\alpha<2, T>0$, and $A$ is a closed linear operator defined on $X$ which generates the $(\alpha, 1)$-resolvent family $\left\{S_{\alpha, 1}(t)\right\}_{t \geq 0}$. The nonlinear function $f:[0, T] \times X \rightarrow X$ is continuous and the nonlocal conditions $p, q: C(I, X) \rightarrow$ $C(I, X)$ are also continuous functions. We recall also that the derivative $D_{t}^{\alpha}$ denotes the Caputo fractional derivative.

The mild solution to problem (45) is given by

$$
\begin{aligned}
u(t)= & S_{\alpha, 1}(t)\left(u_{0}-p(u)\right)+S_{\alpha, 2}(t)\left(u_{1}-q(u)\right) \\
& +\int_{0}^{t} S_{\alpha, \alpha}(t-s) f(s, u(s)) d s, \quad t \in[0, T] .
\end{aligned}
$$

By the uniqueness of the Laplace transform, it is easy to see that the mild solution to fractional nonlocal problem (45) can be written as 


$$
\begin{aligned}
u(t)= & S_{\alpha, 1}(t)\left(u_{0}-p(u)\right) \\
& +\left(g_{1} * S_{\alpha, 1}\right)(t)\left(u_{1}-q(u)\right) \\
& +\int_{0}^{t}\left(g_{\alpha-1} * S_{\alpha, 1}\right)(t-s) f(s, u(s)) d s
\end{aligned}
$$

for all $t \in[0, T]$.

We assume the following:

(H1) The function $f$ satisfies the Carathéodory condition; that is, $f(\cdot, u)$ is strongly measurable for each $u \in X$ and $f(t, \cdot)$ is continuous for each $t \in I:=[0, T]$.

(H2) There exists a continuous function $\mu: I \rightarrow \mathbb{R}_{+}$such that

$\|f(t, u)\| \leq \mu(t)\|u\|, \quad \forall t \in I, u \in C(I, X)$.

(H3) The functions $p, q: C(I, X) \rightarrow C(I, X)$ are continuous and there exist $L_{p}, L_{q}>0$ such that

$$
\begin{gathered}
\|p(u)-p(v)\|<L_{p}\|u-v\|, \\
\|q(u)-q(v)\|<L_{q}\|u-v\|,
\end{gathered}
$$

$$
\forall u, v \in C(I, X)
$$

We have the following existence results.

Theorem 20. Let $1<\alpha<2$. Let $A$ be the generator of an $(\alpha, 1)$-resolvent family $\left\{S_{\alpha, 1}(t)\right\}_{t \geq 0}$ of type $(M, \omega)$. Suppose that $\left(\lambda^{\alpha}-A\right)^{-1}$ is compact for all $\lambda>\omega^{1 / \alpha}$. If $\left(M e^{\omega T} / \omega^{\alpha-1}\right)\|\mu\|_{\infty} T<$ 1 and $\left(M e^{\omega T} L_{p}+(M / \omega) e^{\omega T} L_{q}\right)<1$, then, under assumptions (H1)-(H3), problem (45) has at least one mild solution.

Proof. Let $B_{r}:=\{u \in C(I, X):\|u\| \leq r\}$, where

$$
:=\frac{M e^{\omega T}\left(\left\|u_{0}\right\|+\|p(u)\|\right)+(M / \omega) e^{\omega T}\left(\left\|u_{1}\right\|+\|q(u)\|\right)}{1-\left(M e^{\omega T} / \omega^{\alpha-1}\right)\|\mu\|_{\infty} T} .
$$

On $B_{r}$ we define the operators $\Gamma_{1}, \Gamma_{2}$ by

$$
\begin{aligned}
&\left(\Gamma_{1} u\right)(t):= S_{\alpha, 1}(t)\left[u_{0}-p(u)\right] \\
&+\left(g_{1} * S_{\alpha, 1}\right)(t)\left(u_{1}-q(u)\right), \\
& t \in[0, T], \\
&\left(\Gamma_{2} u\right)(t):=\int_{0}^{t}\left(g_{\alpha-1} * S_{\alpha, 1}\right)(t-s) f(s, u(s)) d s, \\
& t \in[0, T],
\end{aligned}
$$

and $u \in B_{r}$. We shall prove that $\Gamma:=\Gamma_{1}+\Gamma_{2}$ has at least one fixed point by the Krasnoselskii fixed point theorem. We will consider several steps in the proof.
Step 1. We will see that if $u, v \in B_{r}$, then $\Gamma_{1} u+\Gamma_{2} v \in B_{r}$. In fact, by Lemma 12 we have

$$
\begin{aligned}
&\left\|\left(\Gamma_{1} u\right)(t)+\left(\Gamma_{2} v\right)(t)\right\| \\
& \leq\left\|S_{\alpha, 1}(t)\right\|\left\|u_{0}-p(u)\right\| \\
&+\left\|\left(g_{1} * S_{\alpha, 1}\right)(t)\right\|\left\|u_{1}-q(u)\right\| \\
& \quad+\int_{0}^{t}\left\|\left(g_{\alpha-1} * S_{\alpha, 1}\right)(t-s)\right\|\|f(s, v(s))\| d s \\
& \leq M e^{\omega t}\left(\left\|u_{0}\right\|+\|p(u)\|\right)+\frac{M}{\omega} e^{\omega t}\left(\left\|u_{1}\right\|+\|q(u)\|\right) \\
&+\int_{0}^{t}\left\|\left(g_{\alpha-1} * S_{\alpha, 1}\right)(t-s)\right\|\|f(s, v(s))\| d s \\
& \leq M e^{\omega T}\left(\left\|u_{0}\right\|+\|p(u)\|\right) \\
&+\frac{M}{\omega} e^{\omega T}\left(\left\|u_{1}\right\|+\|q(u)\|\right) \\
&+\frac{M}{\omega} e^{\omega T}\left(\left\|u_{1}\right\|+\|q(u)\|\right)+\frac{M r e^{\omega T}}{\omega^{\alpha-1}} \int_{0}^{\alpha-1} e^{\omega(t-s)} \mu \mu \|_{\infty} T \\
& \leq M e^{\omega T}\left(\| e^{\omega T}\left(\left\|u_{0}\right\|+\|p(u)\|\right)\right. \\
&+\frac{M}{\omega} e^{\omega T}\left(\left\|u_{1}\right\|+\|q(u)\|\right) \\
&+\frac{M r}{\omega} e_{0}^{t} e^{-\omega s} \mu(s) d s
\end{aligned}
$$

Hence $\Gamma_{1} u+\Gamma_{2} v \in B_{r}$ for all $u, v \in B_{r}$.

Step 2. $\Gamma_{1}$ is a contraction on $B_{r}$. In fact, if $u, v \in B_{r}$, then

$$
\begin{aligned}
\left\|\Gamma_{1} u(t)-\Gamma_{1} v(t)\right\| \leq & \left\|S_{\alpha, 1}(t)\right\|\|p(u)-p(v)\| \\
& +\left\|\left(g_{1} * S_{\alpha, 1}\right)(t)\right\|\|q(u)-q(v)\| \\
\leq & M e^{\omega t} L_{p}\|u-v\| \\
& +\frac{M}{\omega} e^{\omega t} L_{q}\|u-v\| \\
\leq & \left(M e^{\omega T} L_{p}+\frac{M}{\omega} e^{\omega T} L_{q}\right)\|u-v\| .
\end{aligned}
$$

Since $\left(M e^{\omega T} L_{p}+(M / \omega) e^{\omega T} L_{q}\right)<1$, we conclude that $\Gamma_{1}$ is a contraction. 
Step 3. $\Gamma_{2}$ is completely continuous.

Firstly, we prove that $\Gamma_{2}$ is a continuous operator on $B_{r}$. Let $u_{n}, u \in B_{r}$ such that $u_{n} \rightarrow u$ in $B_{r}$. By Lemma 12 we get

$$
\begin{aligned}
& \left\|\Gamma_{2} u_{n}(t)-\Gamma_{2} u(t)\right\| \leq \int_{0}^{t}\left\|\left(g_{\alpha-1} * S_{\alpha, 1}\right)(t-s)\right\| \\
& \cdot\left\|f\left(s, u_{n}(s)\right)-f(s, u(s))\right\| d s \leq \frac{M e^{\omega t}}{\omega^{\alpha-1}} \\
& \cdot \int_{0}^{t} e^{-\omega s}\left\|f\left(s, u_{n}(s)\right)-f(s, u(s))\right\| d s \leq \frac{M e^{\omega T}}{\omega^{\alpha-1}} \\
& \cdot \int_{0}^{t} \mu(s)\left(\left\|u_{n}(s)\right\|+\|u(s)\|\right) d s \leq \frac{2 r M e^{\omega T}}{\omega^{\alpha-1}} \\
& \cdot \int_{0}^{t} \mu(s) d s .
\end{aligned}
$$

We notice that the function $s \mapsto \mu(s)$ is integrable on $I$. By Lebesgue's dominated convergence theorem, $\int_{0}^{t} \| f\left(s, u_{n}(s)\right)-$ $f(s, u(s)) \| d s \rightarrow 0$ as $n \rightarrow \infty$. Since $u_{n} \rightarrow u$ we obtain that $\Gamma_{2}$ is continuous in $B_{r}$.

Now, we will prove that $\left\{\Gamma_{2} u: u \in B_{r}\right\}$ is relatively compact. By the Ascoli-Arzela theorem, we need to show that the family $\left\{\Gamma_{2} u: u \in B_{r}\right\}$ is uniformly bounded and equicontinuous, and the set $\left\{\Gamma_{2} u(t): u \in B_{r}\right\}$ is relatively compact in $X$ for each $t \in[0, T]$. In fact, for each $u \in B_{r}$ we have (as in Step 3) that $\left\|\Gamma_{2} u\right\| \leq\left(r M e^{\omega T} / \omega^{\alpha-1}\right)\|\mu\|_{\infty}$ and therefore $\left\{\Gamma_{2} u: u \in B_{r}\right\}$ is uniformly bounded.

In order to prove the equicontinuity, let $u \in B_{r}$, and take $0 \leq t_{2}<t_{1} \leq T$. Observe that

$$
\begin{aligned}
& \left\|\Gamma_{2} u\left(t_{1}\right)-\Gamma_{2} u\left(t_{2}\right)\right\| \leq \int_{t_{2}}^{t_{1}} \|\left(g_{\alpha-1} * S_{\alpha, 1}\right)\left(t_{1}-s\right) \\
& \cdot f(s, u(s))\left\|d s+\int_{0}^{t_{2}}\right\|\left(\left(g_{\alpha-1} * S_{\alpha, 1}\right)\left(t_{1}-s\right)\right. \\
& \left.-\left(g_{1} * S_{\alpha, 1}\right)\left(t_{2}-s\right)\right) f(s, u(s)) \| d s:=I_{1} \\
& +I_{2} .
\end{aligned}
$$

Observe that, for $I_{1}$, by Lemma 12 we have

$$
\begin{aligned}
I_{1} & \leq \frac{M e^{\omega T}}{\omega^{\alpha-1}} \int_{t_{2}}^{t_{1}} e^{-\omega s} \mu(s)\|u(s)\| d s \\
& \leq \frac{M r e^{\omega T}}{\omega^{\alpha-1}}\|\mu\|_{\infty}\left(t_{1}-t_{2}\right),
\end{aligned}
$$

and therefore $\lim _{t_{1} \rightarrow t_{2}} I_{1}=0$. For $I_{2}$, we have

$$
\begin{aligned}
I_{2} & \leq \int_{0}^{t_{2}} \|\left(g_{\alpha-1} * S_{\alpha, 1}\right)\left(t_{1}-s\right) \\
& -\left(g_{\alpha-1} * S_{\alpha, 1}\right)\left(t_{2}-s\right)\|\| f(s, u(s)) \| d s \\
& \leq \int_{0}^{t_{2}} \mu(s) \|\left(g_{\alpha-1} * S_{\alpha, 1}\right)\left(t_{1}-s\right) \\
& -\left(g_{\alpha-1} * S_{\alpha, 1}\right)\left(t_{2}-s\right)\|\| u(s) \| d s \leq r \int_{0}^{t_{2}} \mu(s) \\
& \cdot \|\left(g_{\alpha-1} * S_{\alpha, 1}\right)\left(t_{1}-s\right) \\
& -\left(g_{\alpha-1} * S_{\alpha, 1}\right)\left(t_{2}-s\right) \| d s .
\end{aligned}
$$

Observe that

$$
\begin{aligned}
& \mu(\cdot)\left\|\left(g_{\alpha-1} * S_{\alpha, 1}\right)\left(t_{1}-\cdot\right)-\left(g_{\alpha-1} * S_{\alpha, 1}\right)\left(t_{2}-\cdot\right)\right\| \\
& \quad \leq 2 \frac{M e^{\omega T}}{\omega^{\alpha-1}} \mu(\cdot) \in L^{1}(I, \mathbb{R}),
\end{aligned}
$$

and, by Lemma 12, $\left(g_{\alpha-1} * S_{\alpha, 1}\right)(t)=S_{\alpha, \alpha}(t)$ for all $t \geq 0$. Moreover, by Proposition 11 we have that $S_{\alpha, \alpha}(t)$ is norm continuous and therefore if $t_{1} \rightarrow t_{2}$, then $\left(g_{\alpha-1} * S_{\alpha, 1}\right)\left(t_{1}-\right.$ $s)-\left(g_{\alpha-1} * S_{\alpha, 1}\right)\left(t_{2}-s\right) \rightarrow 0$ in $\mathscr{B}(X)$. We obtain by Lebesgue's dominated convergence theorem that $\lim _{t_{1} \rightarrow t_{2}} I_{2}=$ 0 . Therefore, $\left\{\Gamma_{2} u: u \in B_{r}\right\}$ is an equicontinuous family.

Finally, we prove that $H(t):=\left\{\Gamma_{2} u(t): u \in B_{r}\right\}$ is relatively compact in $X$ for each $t \in[0, T]$. Obviously, $H(0)$ is relatively compact in $X$. Now, we take $t>0$. For $0<\varepsilon<t$ we define on $B_{r}$ the operator

$$
\left(\Gamma_{2}^{\varepsilon} u\right)(t):=\int_{0}^{t-\varepsilon}\left(g_{\alpha-1} * S_{\alpha, 1}\right)(t-s) f(s, u(s)) d s .
$$

The hypotheses implies the compactness of $\left(g_{\alpha-1} * S_{\alpha, 1}\right)(t)=$ $S_{\alpha, \alpha}(t)$ for all $t>0$ (by Lemma 12 and Theorem 14) and therefore the set $\mathscr{K}_{\varepsilon}:=\left\{\left(g_{\alpha-1} * S_{\alpha, 1}\right)(t-s) f(s, u(s)): u \in\right.$ $\left.B_{r}, 0 \leq s \leq t-\varepsilon\right\}$ is compact for all $\varepsilon>0$. Then $\overline{\operatorname{conv}\left(\mathscr{K}_{\varepsilon}\right)}$ is also a compact set by Theorem 7. By using the mean-value theorem for the Bochner integrals (see [32, Corollary 8, page 48]), we obtain that

$$
\left(\Gamma_{2}^{\varepsilon} u\right)(t) \in \overline{t \operatorname{conv}\left(\mathscr{K}_{\varepsilon}\right)}, \quad \forall t \in[0, T] .
$$

Therefore, the set $H_{\varepsilon}(t):=\left\{\left(\Gamma_{2}^{\varepsilon} u\right)(t): u \in B_{r}\right\}$ is relatively compact in $X$ for all $\varepsilon>0$. Now, observe that

$$
\begin{aligned}
& \left\|\left(\Gamma_{2} u\right)(t)-\left(\Gamma_{2}^{\varepsilon} u\right)(t)\right\| \\
& \leq \int_{t-\varepsilon}^{t}\left\|\left(g_{\alpha-1} * S_{\alpha, 1}\right)(t-s) f(s, u(s))\right\| d s \\
& \quad \leq \frac{M r e^{\omega T}}{\omega^{\alpha-1}} \int_{t-\varepsilon}^{t} e^{-\omega s} \mu(s) d s .
\end{aligned}
$$

Since the function $s \mapsto e^{-\omega s} \mu(s)$ belongs to $L^{1}\left([t-\varepsilon, t], \mathbb{R}_{+}\right)$, we conclude by the Lebesgue dominated convergence theorem that

$$
\lim _{\varepsilon \rightarrow 0}\left\|\left(\Gamma_{2} u\right)(t)-\left(\Gamma_{2}^{\varepsilon} u\right)(t)\right\|=0 .
$$


Therefore the set $\left\{\Gamma_{2} u(t): u \in B_{r}\right\}$ is relatively compact in $X$ for each $t \in(0, T]$. By the Ascoli-Arzela theorem, the set $\left\{\Gamma_{2} u: u \in B_{r}\right\}$ is relatively compact. We conclude that $\Gamma_{2}$ is a completely continuous operator. Hence, by Krasnoselskii Theorem 8 we have that $\Gamma=\Gamma_{1}+\Gamma_{2}$ has a fixed point on $B_{r}$, which means that nonlocal problem (45) has a mild solution and the proof of the theorem is finished.

The proof of the following result uses the Schauder fixed point theorem. We notice that here we will assume that $S_{\alpha, 1}(t)$ is continuous in the uniform operator topology for all $t>0$. Moreover, we have a weaker condition on the parameters $M$, $\omega$, and $T$.

Theorem 21. Let $1<\alpha<2$. Let $A$ be the generator of an $(\alpha, 1)$ resolvent family $\left\{S_{\alpha, 1}(t)\right\}_{t \geq 0}$ of type $(M, \omega)$. Suppose that $\left(\lambda^{\alpha}-\right.$ $A)^{-1}$ is compact for all $\lambda>\omega^{1 / \alpha}, S_{\alpha, 1}(t)$ is continuous in the uniform operator topology for all $t>0$, and $M e^{\omega T}\|\mu\|_{\infty} T<1$. Then, under assumptions (H1)-(H3), problem (45) has at least one mild solution.

Proof. We define the operator $\Gamma: C(I, X) \rightarrow C(I, X)$ by

$$
\begin{aligned}
(\Gamma u)(t):= & S_{\alpha, 1}(t)\left[u_{0}-p(u)\right] \\
& +\left(g_{1} * S_{\alpha, 1}\right)(t)\left(u_{1}-q(u)\right) \\
& +\int_{0}^{t}\left(g_{\alpha-1} * S_{\alpha, 1}\right)(t-s) f(s, u(s)) d s, \\
& t \in I=[0, T] .
\end{aligned}
$$

Choose

$r$

$$
=\frac{M e^{\omega T}\left(\left\|u_{0}\right\|+\|p(u)\|\right)+(M / \omega) e^{\omega T}\left(\left\|u_{1}\right\|+\|q(u)\|\right)}{1-\left(M e^{\omega T} / \omega^{\alpha-1}\right)\|\mu\|_{\infty} T} .
$$

Let $B_{r}:=\{u \in C(I, X):\|u\| \leq r\}$. We shall prove that $\Gamma$ : $B_{r} \rightarrow B_{r}$ has at least one fixed point by the Schauder fixed point theorem. As in the proof of Theorem 20 it is easy to see that $\Gamma$ sends $B_{r}$ into $B_{r}$, and $\Gamma: B_{r} \rightarrow B_{r}$ is a continuous operator.

We claim that $\left\{\Gamma u: u \in B_{r}\right\}$ is relatively compact.

Indeed, as in the proof of Theorem 20, it is easy to see that $\left\{\Gamma u: u \in B_{r}\right\}$ is uniformly bounded. On the other hand, to see the equicontinuity, let $u \in B_{r}$, and take $t_{1}, t_{2} \in I$ with $0 \leq t_{2}<t_{1} \leq T$. We have

$$
\begin{aligned}
& \left\|\Gamma u\left(t_{1}\right)-\Gamma u\left(t_{2}\right)\right\| \leq \|\left(S_{\alpha, 1}\left(t_{1}\right)-S_{\alpha, 1}\left(t_{2}\right)\right)\left(u_{0}\right. \\
& -p(u))\|+\|\left(\left(g_{1} * S_{\alpha, 1}\right)\left(t_{1}\right)-\left(g_{1} * S_{\alpha, 1}\right)\left(t_{2}\right)\right) \\
& \cdot\left(u_{1}-q(u)\right)\left\|+\int_{t_{2}}^{t_{1}}\right\|\left(g_{\alpha-1} * S_{\alpha, 1}\right)\left(t_{1}-s\right) \\
& \cdot f(s, u(s))\left\|d s+\int_{0}^{t_{2}}\right\|\left(\left(g_{\alpha-1} * S_{\alpha, 1}\right)\left(t_{1}-s\right)\right. \\
& \left.-\left(g_{1} * S_{\alpha, 1}\right)\left(t_{2}-s\right)\right) f(s, u(s)) \| d s:=I_{1}+I_{2} \\
& +I_{3}+I_{4} .
\end{aligned}
$$

Observe that for $I_{1}$ we have

$$
I_{1} \leq\left\|S_{\alpha, 1}\left(t_{1}\right)-S_{\alpha, 1}\left(t_{2}\right)\right\|\left\|\left(u_{0}-g(u)\right)\right\| .
$$

By hypothesis, using the norm continuity of $S_{\alpha, 1}(t)$, we obtain that $\lim _{t_{1} \rightarrow t_{2}} I_{1}=0$.

Lemma 12 implies $\left(g_{1} * S_{\alpha, 1}\right)(t)=S_{\alpha, 2}(t)$ for all $t \geq 0$ and by Proposition 11 we have that $\left(g_{1} * S_{\alpha, 1}\right)(t)$ is continuous in $\mathscr{B}(X)$, and hence

$$
\begin{aligned}
I_{2} & \leq\left\|\left(g_{1} * S_{\alpha, 1}\right)\left(t_{1}\right)-\left(g_{1} * S_{\alpha, 1}\right)\left(t_{2}\right)\right\|\left\|\left(u_{0}-g(u)\right)\right\| \\
& \longrightarrow 0
\end{aligned}
$$

as $t_{1} \rightarrow t_{2}$. On the other hand, $I_{3}, I_{4} \rightarrow 0$ as $t_{1} \rightarrow t_{2}$ as in the proof of Step 3 in Theorem 20. Therefore, the set $\left\{\Gamma u: u \in B_{r}\right\}$ is equicontinuous.

Finally, we will prove that $\left\{\Gamma u(t): u \in B_{r}\right\}$ is relatively compact for all $t \in[0, T]$. Clearly, $\left\{\Gamma u(0): u \in B_{r}\right\}$ is relatively compact. Now, we take $t>0$. For each $0<\varepsilon<t$, we define the operator

$$
\begin{aligned}
& \left(\Gamma^{\varepsilon} u\right)(t) \\
& :=S_{\alpha, 1}(\varepsilon) \int_{0}^{t-\varepsilon}\left(g_{\alpha-1} * S_{\alpha, 1}\right)(t-s-\varepsilon) f(s, u(s)) d s .
\end{aligned}
$$

The hypothesis and Proposition 16 show that $S_{\alpha, 1}(t)$ is compact for all $t>0$ and therefore the set $H_{\mathcal{E}}(t):=\left\{\left(\Gamma_{2}^{\varepsilon} u\right)(t)\right.$ : $\left.u \in B_{r}\right\}$ is relatively compact in $X$ for all $\varepsilon>0$. Now, observe that

$$
\begin{aligned}
& \| S_{\alpha, 1}(\varepsilon) \int_{0}^{t-\varepsilon}\left(g_{\alpha-1} * S_{\alpha, 1}\right)(t-s-\varepsilon) f(s, u(s)) d s \\
& \quad-\int_{0}^{t-\varepsilon}\left(g_{\alpha-1} * S_{\alpha, 1}\right)(t-s) f(s, u(s)) d s \| \\
& \quad \leq r \int_{0}^{t-\varepsilon} \| S_{\alpha, 1}(\varepsilon)\left(g_{\alpha-1} * S_{\alpha, 1}\right)(t-s-\varepsilon) \\
& \quad-\left(g_{\alpha-1} * S_{\alpha, 1}\right)(t-s) \| \mu(s) d s .
\end{aligned}
$$

By Proposition 11, $\left(g_{\alpha-1} * S_{\alpha, 1}\right)(t)$ is norm continuous for all $t>0$ and therefore

$$
\begin{aligned}
& \| S_{\alpha, 1}(\varepsilon)\left(g_{\alpha-1} * S_{\alpha, 1}\right)(t-s-\varepsilon) \\
& \quad-\left(g_{\alpha-1} * S_{\alpha, 1}\right)(t-s) \| \longrightarrow 0, \quad \text { as } \varepsilon \longrightarrow 0 .
\end{aligned}
$$

On the other hand, since

$$
\begin{aligned}
& \left\|S_{\alpha, 1}(\varepsilon)\left(g_{\alpha-1} * S_{\alpha, 1}\right)(t-\cdot-\varepsilon)-\left(g_{\alpha-1} * S_{\alpha, 1}\right)(t-\cdot)\right\| \\
& \quad \leq \frac{M^{2} e^{2 \omega T}}{\omega^{\alpha-1}} e^{-\omega(\cdot+\varepsilon)}+\frac{M e^{\omega T}}{\omega^{\alpha-1}} e^{-\omega .}
\end{aligned}
$$

and the function $s \mapsto\left(M^{2} e^{2 \omega T} / \omega^{\alpha-1}\right) e^{-\omega(s+\varepsilon)}+\left(M e^{\omega T} /\right.$ $\left.\omega^{\alpha-1}\right) e^{-\omega s}$ belongs to $L^{1}\left(I, \mathbb{R}_{+}\right)$, we conclude by the Lebesgue dominated convergence theorem that

$$
\begin{gathered}
\lim _{\varepsilon \rightarrow 0} \| S_{\alpha, 1}(\varepsilon) \int_{0}^{t-\varepsilon}\left(g_{\alpha-1} * S_{\alpha, 1}\right)(t-s-\varepsilon) f(s, u(s)) d s \\
-\int_{0}^{t-\varepsilon}\left(g_{\alpha-1} * S_{\alpha, 1}\right)(t-s) f(s, u(s)) d s \|=0 .
\end{gathered}
$$


As in the proof of [24, Theorem 4.1], we get

$$
\begin{gathered}
\lim _{\varepsilon \rightarrow 0} \| S_{\alpha, 1}(\varepsilon) \int_{0}^{t-\varepsilon}\left(g_{\alpha-1} * S_{\alpha, 1}\right)(t-s-\varepsilon) f(s, u(s)) d s \\
-\int_{0}^{t}\left(g_{\alpha-1} * S_{\alpha, 1}\right)(t-s) f(s, u(s)) d s \|=0,
\end{gathered}
$$

and therefore the set $\left\{\int_{0}^{t}\left(g_{\alpha-1} * S_{\alpha, 1}\right)(t-s) f(s, u(s)) d s: u \in\right.$ $\left.B_{r}\right\}$ is relatively compact for all $t \in(0, T]$. The compactness of $S_{\alpha, 1}(t)$ and $\left(g_{1} * S_{\alpha, 1}\right)(t)=S_{\alpha, 2}(t)$ (by Lemma 12 and Theorem 14) imply that $\left\{\Gamma u(t): u \in B_{r}\right\}$ is relatively compact in $X$ for each $t \in(0, T]$. By the Ascoli-Arzela theorem, the set $\left\{\Gamma u: u \in B_{r}\right\}$ is relatively compact. We conclude that $\Gamma$ is a compact operator on $B_{r}$. Hence, by Schauder Theorem 9 we have that $\Gamma$ has a fixed point on $B_{r}$ and therefore nonlocal problem (45) has a mild solution.

Remark 22. We notice that the norm continuity of $S_{\alpha, 1}(t)$ for $0<\alpha<1$ and $t>0$ follows, for example, if $\left\{S_{\alpha, 1}(t)\right\}_{t \geq 0}$ is analytic (see [24, Lemma 3.8]) or if $A$ is an almost sectorial operator (see [23, Theorem 3.2]).

Now, we consider the nonlocal problem for the Caputo fractional derivative

$$
\begin{aligned}
D_{t}^{\alpha} u(t) & =A u(t)+f(t, u(t)), \quad t \in I:=[0, T], \\
u(0)+p(u) & =u_{0}
\end{aligned}
$$

$u_{0} \in X, 1 / 2<\alpha<1, T>0$, and $A$ is a closed linear operator defined on $X$ which generates the $(\alpha, \alpha)$-resolvent family $\left\{S_{\alpha, \alpha}(t)\right\}_{t \geq 0}$.

The mild solution to problem (74) is given by

$$
\begin{aligned}
u(t)= & S_{\alpha, 1}(t)\left(u_{0}-p(u)\right) \\
& +\int_{0}^{t} S_{\alpha, \alpha}(t-s) f(s, u(s)) d s, \quad t \in[0, T] .
\end{aligned}
$$

It is easy to see (by using the uniqueness of the Laplace transform) that the mild solution to problem (74) can be also written as

$$
\begin{aligned}
u(t)= & \left(g_{1-\alpha} * S_{\alpha, \alpha}\right)(t)\left(u_{0}-p(u)\right) \\
& +\int_{0}^{t} S_{\alpha, \alpha}(t-s) f(s, u(s)) d s, \quad t \in[0, T] .
\end{aligned}
$$

The proof of the following result follows similarly to Theorem 20 and therefore we omit it.

Theorem 23. Let $1 / 2<\alpha<1$. Let $A$ be the generator of an $(\alpha, \alpha)$-resolvent family $\left\{S_{\alpha, \alpha}(t)\right\}_{t \geq 0}$ of type $(M, \omega)$. Suppose that $\left(\lambda^{\alpha}-A\right)^{-1}$ is compact for all $\lambda>\omega^{1 / \alpha}$, and $S_{\alpha, \alpha}(t)$ is continuous in the uniform operator topology for all $t>0$. If $M e^{\omega T}\|\mu\|_{\infty} T<$ 1 and $\left(M / \omega^{1-\alpha}\right) e^{\omega T} L_{p}<1$, then, under assumptions (H1)(H3), problem (74) has at least one mild solution.

\section{Nonlocal Fractional Cauchy Problems: The Riemann-Liouville Case}

In this section we consider the nonlocal problem for the Riemann-Liouville fractional derivative

$$
D^{\alpha} u(t)=A u(t)+f(t, u(t)),
$$

$$
t \in[0, T],
$$

$$
\begin{aligned}
& \left(g_{2-\alpha} * u\right)(0)+p(u)=u_{0}, \\
& \left(g_{2-\alpha} * u\right)^{\prime}(0)+q(u)=u_{1},
\end{aligned}
$$

where $u_{0}, u_{1} \in X, 1<\alpha<2$, and $A$ is a closed linear operator defined on $X$. Assume that $A$ generates an $(\alpha, \alpha-1)$-resolvent family given by $\left\{S_{\alpha, \alpha-1}(t)\right\}_{t \geq 0}$. Taking Laplace transform in (77) we obtain by (14) that

$$
\begin{aligned}
u(t)= & S_{\alpha, \alpha-1}(t)\left(u_{0}-p(u)\right)+S_{\alpha, \alpha}(t)\left(u_{1}-q(u)\right) \\
& +\int_{0}^{t} S_{\alpha, \alpha}(t-s) f(s, u(s)) d s, \quad t \in[0, T] .
\end{aligned}
$$

The uniqueness of the Laplace transform implies that the mild solution $u$ to problem (77) is also given by

$$
\begin{aligned}
u(t)= & S_{\alpha, \alpha-1}(t)\left(u_{0}-p(u)\right) \\
& +\left(g_{1} * S_{\alpha, \alpha-1}\right)(t)\left(u_{1}-q(u)\right) \\
& +\int_{0}^{t}\left(g_{1} * S_{\alpha, \alpha-1}\right)(t-s) f(s, u(s)) d s
\end{aligned}
$$

for all $t \in[0, T]$.

Theorem 24. Let $1<\alpha<2$. Let $A$ be the generator of an $(\alpha, \alpha-1)$-resolvent family $\left\{S_{\alpha, \alpha-1}(t)\right\}_{t \geq 0}$ of type $(M, \omega)$. Assume that the resolvent $\left(\lambda^{\alpha}-A\right)^{-1}$ is compact for all $\lambda>\omega^{1 / \alpha}$. If $\left(M e^{\omega T} L_{p}+(M / \omega) e^{\omega T} L_{q}\right)<1$ and $\left(M e^{\omega T} / \omega\right)\|\mu\|_{\infty} T<1$, then, under assumptions (H1)-(H3), problem (77) has at least one mild solution.

Proof. Let $B_{r}:=\{u \in C(I, X):\|u\| \leq r\}$, where

$$
:=\frac{M e^{\omega T}\left(\left\|u_{0}\right\|+\|p(u)\|\right)+(M / \omega) e^{\omega T}\left(\left\|u_{1}\right\|+\|q(u)\|\right)}{1-\left(M e^{\omega T} / \omega\right)\|\mu\|_{\infty} T} .
$$

On $B_{r}$ we define the operators $\Gamma_{1}, \Gamma_{2}$ by

$$
\begin{aligned}
\left(\Gamma_{1} u\right)(t):= & S_{\alpha, \alpha-1}(t)\left[u_{0}-p(u)\right] \\
& +\left(g_{1} * S_{\alpha, \alpha-1}\right)(t)\left(u_{1}-q(u)\right), \\
t \in[0, T], & t \in(s, u(s)) d s,
\end{aligned}
$$


and $u \in B_{r}$. We shall prove that $\Gamma:=\Gamma_{1}+\Gamma_{2}$ has at least one fixed point by the Krasnoselskii fixed point theorem. We will consider several steps in the proof.

Step 1. We will see that if $u, v \in B_{r}$, then $\Gamma_{1} u+\Gamma_{2} v \in B_{r}$. In fact, by Lemma 12 we have

$$
\begin{aligned}
\left\|\left(\Gamma_{1} u\right)(t)+\left(\Gamma_{2} v\right)(t)\right\| & \left\|S_{\alpha, \alpha-1}(t)\right\|\left\|u_{0}-p(u)\right\| \\
& +\left\|\left(g_{1} * S_{\alpha, \alpha-1}\right)(t)\right\|\left\|u_{1}-q(u)\right\| \\
& +\int_{0}^{t}\left\|\left(g_{1} * S_{\alpha, \alpha-1}\right)(t-s)\right\|\|f(s, v(s))\| d s \\
\leq & M e^{\omega t}\left(\left\|u_{0}\right\|+\|p(u)\|\right)+\frac{M}{\omega} e^{\omega t}\left(\left\|u_{1}\right\|+\|q(u)\|\right) \\
& +\frac{M r e^{\omega t}}{\omega} \int_{0}^{t} e^{-\omega s} \mu(s) d s \\
\leq & M e^{\omega T}\left(\left\|u_{0}\right\|+\|p(u)\|\right) \\
& +\frac{M}{\omega} e^{\omega T}\left(\left\|u_{1}\right\|+\|q(u)\|\right)+\frac{M r e^{\omega T}}{\omega}\|\mu\|_{\infty} T \\
= & r .
\end{aligned}
$$

Hence $\Gamma_{1} u+\Gamma_{2} v \in B_{r}$ for all $u, v \in B_{r}$.

Step 2. $\Gamma_{1}$ is a contraction on $B_{r}$. In fact, if $u, v \in B_{r}$, then

$$
\begin{aligned}
\left\|\Gamma_{1} u(t)-\Gamma_{1} v(t)\right\| & \\
\leq & \left\|S_{\alpha, \alpha-1}(t)\right\|\|p(u)-p(v)\| \\
& +\left\|\left(g_{1} * S_{\alpha, \alpha-1}\right)(t)\right\|\|q(u)-q(v)\| \\
\leq & M e^{\omega t} L_{p}\|u-v\|+\frac{M}{\omega} e^{\omega t} L_{q}\|u-v\| \\
\leq & \left(M e^{\omega T} L_{p}+\frac{M}{\omega} e^{\omega T} L_{q}\right)\|u-v\| .
\end{aligned}
$$

Since $\left(M e^{\omega T} L_{p}+(M / \omega) e^{\omega T} L_{q}\right)<1$, we conclude that $\Gamma_{1}$ is a contraction.

Step 3. $\Gamma_{2}$ is completely continuous.

As in the proof of Theorem 20 it is easy to see that $\Gamma_{2}$ is a continuous operator and the set $\left\{\Gamma_{2} u: u \in B_{r}\right\}$ is uniformly bounded.

To prove the equicontinuity, let $u \in B_{r}$, and take $0 \leq t_{2}<$ $t_{1} \leq T$. Observe that

$$
\begin{aligned}
& \left\|\Gamma_{2} u\left(t_{1}\right)-\Gamma_{2} u\left(t_{2}\right)\right\| \leq \int_{t_{2}}^{t_{1}} \|\left(g_{1} * S_{\alpha, \alpha-1}\right)\left(t_{1}-s\right) \\
& \cdot f(s, u(s))\left\|d s+\int_{0}^{t_{2}}\right\|\left(\left(g_{1} * S_{\alpha, \alpha-1}\right)\left(t_{1}-s\right)\right. \\
& \left.\quad-\left(g_{1} * S_{\alpha, \alpha-1}\right)\left(t_{2}-s\right)\right) f(s, u(s)) \| d s:=I_{1} \\
& \quad+I_{2} .
\end{aligned}
$$

To estimate $I_{1}$ we notice that

$$
\begin{aligned}
I_{1} & \leq \frac{M e^{\omega T}}{\omega} \int_{t_{2}}^{t_{1}} e^{-\omega s} \mu(s)\|u(s)\| d s \\
& \leq \frac{M r e^{\omega T}}{\omega}\|\mu\|_{\infty}\left(t_{1}-t_{2}\right),
\end{aligned}
$$

and therefore $\lim _{t_{1} \rightarrow t_{2}} I_{1}=0$. For $I_{2}$ we have

$$
\begin{aligned}
I_{2} & \leq \int_{0}^{t_{2}} \|\left(g_{1} * S_{\alpha, \alpha-1}\right)\left(t_{1}-s\right) \\
& -\left(g_{1} * S_{\alpha, \alpha-1}\right)\left(t_{2}-s\right)\|\| f(s, u(s)) \| d s \\
& \leq \int_{0}^{t_{2}} \mu(s) \|\left(g_{1} * S_{\alpha, \alpha-1}\right)\left(t_{1}-s\right) \\
& -\left(g_{1} * S_{\alpha, \alpha-1}\right)\left(t_{2}-s\right)\|\| u(s) \| d s \leq r \int_{0}^{t_{2}} \mu(s) \\
& \cdot \|\left(g_{1} * S_{\alpha, \alpha-1}\right)\left(t_{1}-s\right) \\
& -\left(g_{1} * S_{\alpha, \alpha-1}\right)\left(t_{2}-s\right) \| d s .
\end{aligned}
$$

Observe that

$$
\begin{aligned}
& \mu(\cdot)\left\|\left(g_{1} * S_{\alpha, \alpha-1}\right)\left(t_{1}-\cdot\right)-\left(g_{1} * S_{\alpha, \alpha-1}\right)\left(t_{2}-\cdot\right)\right\| \\
& \leq 2 \frac{M e^{\omega T}}{\omega} \mu(\cdot) \in L^{1}(I, \mathbb{R}),
\end{aligned}
$$

and, by Lemma 12, $\left(g_{1} * S_{\alpha, \alpha-1}\right)(t)=S_{\alpha, \alpha}(t)$ for all $t \geq 0$. Moreover, by Proposition 11 we have that $S_{\alpha, \alpha}(t)$ is norm continuous and therefore if $t_{1} \rightarrow t_{2}$, then $\left(g_{1} * S_{\alpha, \alpha-1}\right)\left(t_{1}-\right.$ $s)-\left(g_{1} * S_{\alpha, \alpha-1}\right)\left(t_{2}-s\right) \rightarrow 0$ in $\mathscr{B}(X)$. We obtain by Lebesgue's dominated convergence theorem that $\lim _{t_{1} \rightarrow t_{2}} I_{2}=$ 0 . Therefore, $\left\{\Gamma_{2} u: u \in B_{r}\right\}$ is an equicontinuous family.

Finally, the compactness of $\left(g_{1} * S_{\alpha, \alpha-1}\right)(t)=S_{\alpha, \alpha}(t)$ for all $t>0$ (by Lemma 12 and Theorem 14) implies that $\left\{\Gamma_{2} u(t)\right.$ : $\left.u \in B_{r}\right\}$ is relatively compact in $X$ for each $t \in[0, T]$ (as in the proof of Theorem 20). We conclude that $\Gamma_{2}$ is a completely continuous operator and, by the Krasnoselskii theorem, the operator $\Gamma=\Gamma_{1}+\Gamma_{2}$ has a fixed point on $B_{r}$, which means that nonlocal problem (77) has at least one mild solution.

In the next result, we consider a weaker condition on the parameters $M, \omega$, and $T$. However, we need to assume here the norm continuity of $S_{\alpha, \alpha-1}(t)$ for $3 / 2<\alpha<2$.

Theorem 25. Let $3 / 2<\alpha<2$. Let $A$ be the generator of an $(\alpha, \alpha-1)$-resolvent family $\left\{S_{\alpha, \alpha-1}(t)\right\}_{t \geq 0}$ of type $(M, \omega)$. Assume that $\left(\lambda^{\alpha}-A\right)^{-1}$ is compact for all $\lambda>\omega^{1 / \alpha}$ and $S_{\alpha, \alpha-1}(t)$ is continuous in the uniform operator topology for all $t>0$. If $\left(M e^{\omega T} / \omega\right)\|\mu\|_{\infty} T<1$, then, under assumptions (H1)-(H3), problem (77) has at least one mild solution. 
Proof. On $B_{r}$ we define the operator

$$
\begin{aligned}
\Gamma u(t):= & S_{\alpha, \alpha-1}(t)\left(u_{0}-p(u)\right) \\
& +\left(g_{1} * S_{\alpha, \alpha-1}\right)(t)\left(u_{1}-q(u)\right) \\
& +\int_{0}^{t}\left(g_{1} * S_{\alpha, \alpha-1}\right)(t-s) f(s, u(s)) d s,
\end{aligned}
$$

where $t \in[0, T]$ and

$$
:=\frac{M e^{\omega T}\left(\left\|u_{0}\right\|+\|p(u)\|\right)+(M / \omega) e^{\omega T}\left(\left\|u_{1}\right\|+\|q(u)\|\right)}{1-\left(M e^{\omega T} / \omega\right)\|\mu\|_{\infty} T} .
$$

The proof follows the same lines of Theorem 21 . We give here only the details on the relative compactness of $\left\{\Gamma_{2} u(t): u \in\right.$ $\left.B_{r}\right\}$ in $X$ for each $t \in[0, T]$. Theorem 14 implies that $\left(g_{1} *\right.$ $\left.S_{\alpha, \alpha-1}\right)(t)=S_{\alpha, \alpha}(t)$ is compact for all $t>0$ and therefore the set $\left\{\int_{0}^{t}\left(g_{1} * S_{\alpha, \alpha-1}\right)(t-s) f(s, u(s)) d s: u \in B_{r}\right\}$ is relatively compact for all $t \in[0, T]$ (as in the proof of Theorem 20). On the other hand, the hypothesis and Proposition 17 imply that $S_{\alpha, \alpha-1}(t)$ is compact for all $t>0$ and thus the set $\{\Gamma u(t): u \in$ $\left.B_{r}\right\}$ is relatively compact for all $t \in[0, T]$. The existence of a fixed point to $\Gamma$, and therefore of a mild solution to problem (77), follows from the Schauder theorem.

Now we discuss the existence of mild solutions to the nonlocal fractional Cauchy problem for the Riemann-Liouville fractional derivative in case $0<\alpha<1$ :

$$
\begin{aligned}
& D^{\alpha} u(t)=A u(t)+f(t, u(t)), \\
& t \in[0, T], \\
&\left(g_{1-\alpha} * u\right)(0)+p(u)=u_{0},
\end{aligned}
$$

where $u_{0} \in X$ and $A$ is a closed linear operator defined on $X$. We assume that $A$ generates an $(\alpha, \alpha)$-resolvent family given by $\left\{S_{\alpha, \alpha}(t)\right\}_{t \geq 0}$. By using the Laplace transform in (90), it is easy to see that

$$
\begin{aligned}
u(t)= & S_{\alpha, \alpha}(t)\left(u_{0}-p(u)\right) \\
& +\int_{0}^{t} S_{\alpha, \alpha}(t-s) f(s, u(s)) d s, \quad t \in[0, T] .
\end{aligned}
$$

Theorem 26. Let $1 / 2<\alpha<1$. Let $A$ be the generator of an $(\alpha, \alpha)$-resolvent family $\left\{S_{\alpha, \alpha}(t)\right\}_{t \geq 0}$ of type $(M, \omega)$. Assume that $\left(\lambda^{\alpha}-A\right)^{-1}$ is compact for all $\lambda>\omega^{1 / \alpha}$, and $S_{\alpha, \alpha}(t)$ is continuous in the uniform operator topology for all $t>0$. If $M e^{\omega T}\|\mu\|_{\infty} T<1$ and $M e^{\omega T} L_{p}<1$, then, under assumptions (H1)-(H3), problem (90) has at least one mild solution.

Proof. Let

$$
r:=\frac{M e^{\omega T}\left(\left\|u_{0}\right\|+\|p(u)\|\right)}{1-M e^{\omega T}\|\mu\|_{\infty} T}
$$

If we define on $B_{r}$ the operators $\Gamma_{1}, \Gamma_{2}$ by

$$
\begin{aligned}
& \left(\Gamma_{1} u\right)(t):=S_{\alpha, \alpha}(t)\left[u_{0}-p(u)\right], \quad t \in[0, T], \\
& \left(\Gamma_{2} u\right)(t):=\int_{0}^{t} S_{\alpha, \alpha}(t-s) f(s, u(s)) d s, \quad t \in[0, T],
\end{aligned}
$$

for $u \in B_{r}$, then, as in the proof of the previous theorems, it is easy to see that if $u, v \in B_{r}$, then $\Gamma_{1} u+\Gamma_{2} v \in B_{r}$, and $\Gamma_{1}$ is a contraction on $B_{r}$. Moreover, $\Gamma_{2}$ is continuous on $B_{r}$, $\left\{\Gamma_{2} u: u \in B_{r}\right\}$ is uniformly bounded, and $\left\{\Gamma_{2} u: u \in B_{r}\right\}$ is an equicontinuous family. Finally, by the compactness of $S_{\alpha, \alpha}(t)$ (see Proposition 18) and by using a similar method as we did in the proof of Theorem 20 (Step 3), we prove that $H(t):=$ $\left\{\Gamma_{2} u(t): u \in B_{r}\right\}$ is relatively compact in $X$ for each $t \in[0, T]$. Thus, by the Ascoli-Arzela theorem, the set $\left\{\Gamma_{2} u: u \in B_{r}\right\}$ is relatively compact and hence $\Gamma_{2}$ is a completely continuous operator. By the Krasnoselskii theorem, we conclude that $\Gamma=$ $\Gamma_{1}+\Gamma_{2}$ has a fixed point on $B_{r}$, and therefore nonlocal problem (90) has at least one mild solution.

\section{Applications}

In this section, we give some applications. As consequence of the previous results, we have the following results.

Consider the semilinear problem

$$
\begin{aligned}
D_{t}^{\alpha} u(t)=A u(t)+J^{2-\alpha} f(t, u(t)), & \\
& t \in I:=[0, T],
\end{aligned}
$$$$
u(0)+p(u)=u_{0}
$$$$
u^{\prime}(0)+q(u)=u_{1} \text {, }
$$

where $u_{0}, u_{1} \in X, J^{2-\alpha}$ denotes the Riemann-Liouville fractional integral operator, $f:[0, T] \times X \rightarrow X$, and $p, q$ : $C(I, X) \rightarrow C(I, X)$ are continuous.

Let $A$ be the generator of an $(\alpha, 1)$-resolvent family $\left\{S_{\alpha, 1}(t)\right\}_{t \geq 0}$. Then it is well known that the mild solution of (94) is defined by means of the variation of constant formula

$$
\begin{aligned}
u(t)= & S_{\alpha, 1}(t)\left[u_{0}-p(u)\right] \\
& +\left(g_{1} * S_{\alpha, 1}\right)(t)\left[u_{1}-q(u)\right] \\
& +\int_{0}^{t}\left(g_{1} * S_{\alpha, 1}\right)(t-s) f(s, u(s)) d s, \quad t \in I .
\end{aligned}
$$

We remark that the case $0<\alpha<1$ was recently studied in [25, Section 4]. On the other hand, we notice that the case $u^{\prime}(0)=$ 0 and $q \equiv 0$ has been recently studied in [33, Section 4$]$ by assuming the relative compactness of the set $\mathscr{K}:=\left\{S_{\alpha, 1}(t-\right.$ s) $f(s, u(s)): u \in C(I, X), 0 \leq s \leq t\}$. Proposition 16 shows that $S_{\alpha, 1}(t)$ is compact for all $t>0$ and by using the LeraySchauder alternative theorem (see Theorem 10) it is easy to prove (as in Theorem 21 and [33, Theorem 4.4]) the following result. We omit the details. 
Theorem 27. Let $1<\alpha<2$. Let $A$ be the generator of an $(\alpha, 1)$-resolvent family $\left\{S_{\alpha, 1}(t)\right\}_{t \geq 0}$ of type $(M, \omega)$. Suppose that $\left(\lambda^{\alpha}-A\right)^{-1}$ is compact for all $\lambda>\omega^{1 / \alpha}$, and $S_{\alpha, 1}(t)$ is continuous in the uniform operator topology for all $t>0$. Then, under assumptions (H1)-(H3), problem (94) has at least one mild solution.

Now, we consider the Riemann-Liouville fractional Cauchy problem

$$
\begin{aligned}
D^{\alpha} u(t) & =A u(t)+J^{2-\alpha} f(t, u(t)), \\
t \in[0, T], & \\
\left(g_{2-\alpha} * u\right)(0)+p(u) & =u_{0}, \\
\left(g_{2-\alpha} * u\right)^{\prime}(0)+q(u) & =u_{1},
\end{aligned}
$$

where $u_{0}, u_{1} \in X, 1<\alpha<2$, and $A$ is a closed linear operator defined on $X$. Assume that $A$ generates an $(\alpha, \alpha-1)$-resolvent family given by $\left\{S_{\alpha, \alpha-1}(t)\right\}_{t \geq 0}$. The mild solution to problem (96) is given by

$$
\begin{aligned}
u(t)= & S_{\alpha, \alpha-1}(t)\left(u_{0}-p(u)\right)+S_{\alpha, \alpha}(t)\left(u_{1}-q(u)\right) \\
& +\int_{0}^{t} S_{\alpha, 2}(t-s) f(s, u(s)) d s, \quad t \in[0, T],
\end{aligned}
$$

which is equivalent (by the uniqueness of the Laplace transform) to

$$
\begin{aligned}
u(t)= & S_{\alpha, \alpha-1}(t)\left(u_{0}-p(u)\right) \\
& +\left(g_{1} * S_{\alpha, \alpha-1}\right)(t)\left(u_{1}-q(u)\right) \\
& +\int_{0}^{t}\left(g_{3-\alpha} * S_{\alpha, \alpha-1}\right)(t-s) f(s, u(s)) d s
\end{aligned}
$$

for all $t \in[0, T]$.

Proposition 17 shows that $S_{\alpha, \alpha-1}(t)$ is compact for all $t>$ 0 (and $3 / 2<\alpha<2$ ) and by using the Leray-Schauder alternative theorem it is easy to prove (as in Theorem 25 and [33, Theorem 4.4] and [25, Theorem 4.1]) the following existence result. We omit the proof.

Theorem 28. Let $3 / 2<\alpha<2$. Let $A$ be the generator of an $(\alpha, \alpha-1)$-resolvent family $\left\{S_{\alpha, \alpha-1}(t)\right\}_{t \geq 0}$ of type $(M, \omega)$. Assume that the resolvent $\left(\lambda^{\alpha}-A\right)^{-1}$ is compact for all $\lambda>\omega^{1 / \alpha}$ and $S_{\alpha, \alpha-1}(t)$ is continuous in the uniform operator topology for all $t>0$. Then, under assumptions (H1)-(H3), problem (96) has at least one mild solution.
We end this section with an example.

Example 29. Consider the following problem:

$$
\begin{aligned}
D_{t}^{\alpha} u(t, x) & =\frac{\partial^{2}}{\partial x^{2}} u(t, x)+f(t, u(t, x)), \\
(t, x) \in[0,1] \times[0, \pi], & \\
u(t, 0) & =u(t, \pi)=0, \quad t \in[0,1], \\
u(0, x)+\sum_{k=1}^{n} a_{k} u(t, x) & =u_{0}(x), \quad x \in[0, \pi],
\end{aligned}
$$

where $1 / 2<\alpha<1, a_{k} \in \mathbb{R}$, and $n \in \mathbb{N}$. Let $X=L^{2}([0, \pi])$ and consider the operator $A: D(A) \subset X \rightarrow X$ defined by $D(A):=\left\{v \in X: v \in H^{2}([0, \pi]), v(0)=v(\pi)\right\}$ and, for $u \in D(A), A u:=\partial^{2} u / \partial x^{2}$.

It is well known that $A$ generates a compact and analytic (and hence norm continuous for all $t>0$ ) $C_{0}$-semigroup $\{T(t)\}_{t \geq 0}$ on $X$ such that $\|T(t)\| \leq 1$ for all $t \geq 0$. Since $A$ generates a $C_{0}$-semigroup, that is, an $(1,1)$-resolvent family, we obtain by [29, Corollary 14 and Theorem 3] that $A$ generates the $(\alpha, \alpha)$-resolvent family $\left\{S_{\alpha, \alpha}(t)\right\}_{t \geq 0}$ defined by

$$
S_{\alpha, \alpha}(t) x:=\int_{0}^{\infty} \varphi_{\alpha, 0}(t, s) T(s) x d s, \quad t>0, x \in X,
$$

where $\varphi_{\alpha, 0}$ is the stable Lévy process of order $\alpha$ defined by (22). Since $T(t)$ is norm continuous, it is easy to see that $S_{\alpha, \alpha}(t)$ is norm continuous for all $t>0$ and the positivity of $\varphi_{\alpha, 0}$ (see [29, Theorem 3]) implies that $S_{\alpha, \alpha}(t)$ is of type $(1,1)$. On the other hand, the compactness of $T(t)$ implies that $\left(\lambda^{\alpha}-A\right)$ is compact.

We notice that problem (99) can be written in the abstract form of (74). Define the functions $f:[0,1] \times D(A) \rightarrow X$ and $p: D(A) \rightarrow X$ by

$$
\begin{aligned}
f(t, u(t, x)) & :=\frac{e^{-t} u(t, x)}{(4+t)(1+u(t, x))}, \\
p(u)(x): & =\sum_{k=1}^{n} a_{k} u(t, x) .
\end{aligned}
$$

Assume that $\sum_{k=1}^{n}\left|a_{k}\right|<1 / 4$. We observe also that in this case we have $\mu(t)=e^{-t} /(4+t), T=M=\omega=1$, and $L_{p}=\|\mu\|_{\infty}=$ $1 / 4$ (see Theorem 23).

It is easy to check assumptions (H1)-(H3) and the hypotheses in Theorem 23 and therefore problem (99) has a mild solution.

Analogously, we can consider the Riemann-Liouville case

$$
\begin{aligned}
& D^{\alpha} u(t, x)=\frac{\partial^{2}}{\partial x^{2}} u(t, x)+f(t, u(t, x)), \\
& \qquad(t, x) \in[0,1] \times[0, \pi], \\
& u(t, 0)=u(t, \pi)=0, \quad t \in[0,1], \\
& \left(g_{1-\alpha} * u\right)(0, x)+\sum_{k=1}^{n} a_{k} u(t, x)=u_{0}(x),
\end{aligned}
$$


Under the same assumptions, we have by Theorem 26 that problem (102) has a mild solution.

6.1. Conclusions. In this paper, we obtain conditions implying the compactness of the family $\left\{S_{\alpha, \beta}(t)\right\}_{t \geq 0}$. As a consequence, we obtain several results on the existence of mild solutions to nonlocal fractional Cauchy problems to the Caputo and Riemann-Liouville fractional derivatives.

\section{Competing Interests}

The author declares that there are no competing interests.

\section{Acknowledgments}

This research was partially supported by FONDECYT Grant no. 11130619.

\section{References}

[1] S. Aizicovici and M. McKibben, "Existence results for a class of abstract nonlocal Cauchy problems," Nonlinear Analysis: Theory, Methods \& Applications, vol. 39, no. 5, pp. 649-668, 2000.

[2] L. Byszewski, "Theorems about the existence and uniqueness of solutions of a semilinear evolution nonlocal Cauchy problem," Journal of Mathematical Analysis and Applications, vol. 162, no. 2, pp. 494-505, 1991.

[3] K. Deng, "Exponential decay of solutions of semilinear parabolic equations with nonlocal initial conditions," Journal of Mathematical Analysis and Applications, vol. 179, no. 2, pp. 630637, 1993.

[4] X. Fu and K. Ezzinbi, "Existence of solutions for neutral functional differential evolution equations with nonlocal conditions," Nonlinear Analysis: Theory, Methods \& Applications, vol. 54, no. 2, pp. 215-227, 2003.

[5] B. Ahmad, J. J. Nieto, A. Alsaedi, and H. Al-Hutami, "Existence of solutions for nonlinear fractional $q$-difference integral equations with two fractional orders and nonlocal four-point boundary conditions," Journal of the Franklin Institute, vol. 351, no. 5, pp. 2890-2909, 2014.

[6] B. de Andrade, C. Cuevas, and H. Soto, "On fractional heat equations with non-local initial conditions," Proceedings of the Edinburgh Mathematical Society Series II, vol. 59, no. 1, pp. 6576, 2016.

[7] A. Debbouche, D. Baleanu, and R. P. Agarwal, "Nonlocal nonlinear integrodifferential equations of fractional orders," Boundary Value Problems, vol. 2012, no. 78, 10 pages, 2012.

[8] G. M. N’Guérékata, “A Cauchy problem for some fractional abstract differential equation with non local conditions," Nonlinear Analysis: Theory, Methods \& Applications, vol. 70, no. 5, pp. 1873-1876, 2009.

[9] G. M. Mophou and G. M. N’Guérékata, "Existence of the mild solution for some fractional differential equations with nonlocal conditions," Semigroup Forum, vol. 79, no. 2, pp. 315-322, 2009.

[10] Z. Liu and X. Li, "Approximate controllability of fractional evolution systems with Riemann-Liouville fractional derivatives," SIAM Journal on Control and Optimization, vol. 53, no. 4, pp. 1920-1933, 2015.
[11] D. Nazari and S. Shahmorad, "Application of the fractional differential transform method to fractional-order integrodifferential equations with nonlocal boundary conditions," Journal of Computational and Applied Mathematics, vol. 234, no. 3, pp. 883-891, 2010.

[12] X.-B. Shu and Q. Wang, "The existence and uniqueness of mild solutions for fractional differential equations with nonlocal conditions of order $1<\alpha<2$," Computers \& Mathematics with Applications, vol. 64, no. 6, pp. 2100-2110, 2012.

[13] J. Wang, Z. Fan, and Y. Zhou, "Nonlocal controllability of semilinear dynamic systems with fractional derivative in Banach spaces," Journal of Optimization Theory and Applications, vol. 154, no. 1, pp. 292-302, 2012.

[14] J. Wang, A. G. Ibrahim, and M. Fečkan, "Nonlocal impulsive fractional differential inclusions with fractional sectorial operators on Banach spaces," Applied Mathematics and Computation, vol. 257, pp. 103-118, 2015.

[15] R.-N. Wang, T.-J. Xiao, and J. Liang, "A note on the fractional Cauchy problems with nonlocal initial conditions," Applied Mathematics Letters, vol. 24, no. 8, pp. 1435-1442, 2011.

[16] Z. Yan, "Existence of solutions for nonlocal impulsive partial functional integrodifferential equations via fractional operators," Journal of Computational and Applied Mathematics, vol. 235, no. 8, pp. 2252-2262, 2011.

[17] Y. Zhou and F. Jiao, "Nonlocal Cauchy problem for fractional evolution equations," Nonlinear Analysis: Real World Applications, vol. 11, no. 5, pp. 4465-4475, 2010.

[18] Y. Zhou and F. Jiao, "Existence of mild solutions for fractional neutral evolution equations," Computers \& Mathematics with Applications, vol. 59, no. 3, pp. 1063-1077, 2010.

[19] X. Liu, Z. Liu, and M. Bin, "The solvability and optimal controls for some fractional impulsive equations of order $1<\alpha<2$," Abstract and Applied Analysis, vol. 2014, Article ID 142067, 9 pages, 2014.

[20] A. Pazy, Semigroups of Linear Operators and Applications to Partial Differential Equations, vol. 44 of Applied Mathematical Sciences, Springer, New York, NY, USA, 1983.

[21] C. Travis and G. Webb, "Compactness, regularity, and uniform continuity properties of strongly continuous cosine families," Houston Journal of Mathematics, vol. 3, no. 4, pp. 555-567, 1977.

[22] J. Prüss, "Positivity and regularity of hyperbolic Volterra equations in Banach spaces," Mathematische Annalen, vol. 279, no. 2, pp. 317-344, 1987.

[23] R.-N. Wang, D.-H. Chen, and T.-J. Xiao, "Abstract fractional Cauchy problems with almost sectorial operators," Journal of Differential Equations, vol. 252, no. 1, pp. 202-235, 2012.

[24] Z. Fan, "Characterization of compactness for resolvents and its applications," Applied Mathematics and Computation, vol. 232, pp. 60-67, 2014.

[25] C. Lizama, A. Pereira, and R. Ponce, "On the compactness of fractional resolvent operator functions," Semigroup Forum, 2016.

[26] C. Lizama, "Regularized solutions for abstract Volterra equations," Journal of Mathematical Analysis and Applications, vol. 243, no. 2, pp. 278-292, 2000.

[27] K. Li, J. Peng, and J. Jia, "Cauchy problems for fractional differential equations with Riemann-Liouville fractional derivatives," Journal of Functional Analysis, vol. 263, no. 2, pp. 476-510, 2012.

[28] C. Lizama and F. Poblete, "On a functional equation associated with $(a, k)$-regularized resolvent families," Abstract and Applied Analysis, vol. 2012, Article ID 495487, 23 pages, 2012. 
[29] L. Abadias and P. J. Miana, "A subordination principle on Wright functions and regularized resolvent families," Journal of Function Spaces, vol. 2015, Article ID 158145, 9 pages, 2015.

[30] L. W. Weis, "A generalization of the Vidav-Jörgens perturbation theorem for semigroups and its application to transport theory," Journal of Mathematical Analysis and Applications, vol. 129, no. 1, pp. 6-23, 1988.

[31] M. Haase, "The complex inversion formula revisited," Journal of the Australian Mathematical Society, vol. 84, no. 1, pp. 73-83, 2008.

[32] J. Diestel and J. Uhl, Vector Measures, Mathematical Surveys, No. 15, American Mathematical Society, Providence, RI, USA, 1977.

[33] C. Lizama and G. M. N'Guérékata, "Mild solutions for abstract fractional differential equations," Applicable Analysis, vol. 92, no. 8, pp. 1731-1754, 2013. 


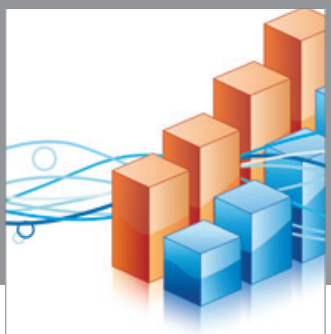

Advances in

Operations Research

vatem alat4

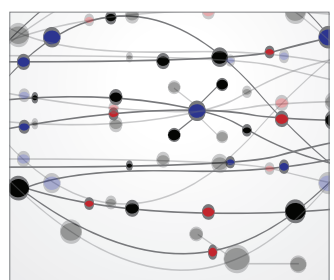

\section{The Scientific} World Journal
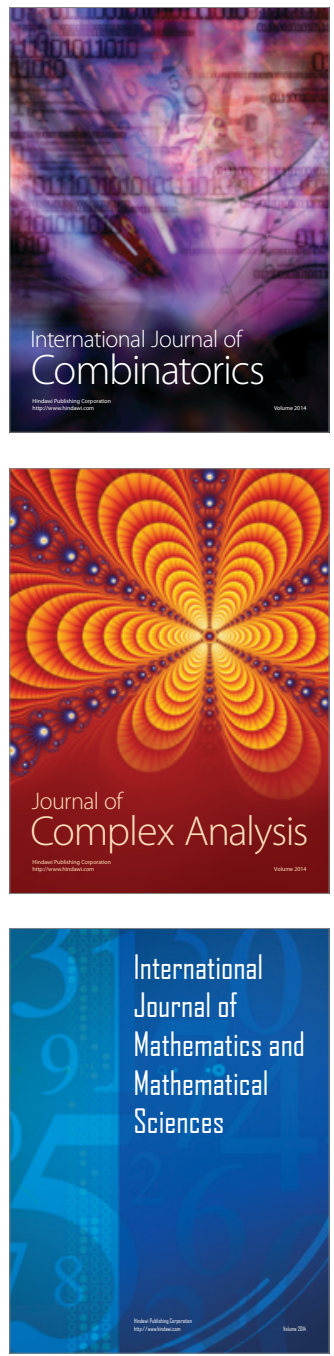
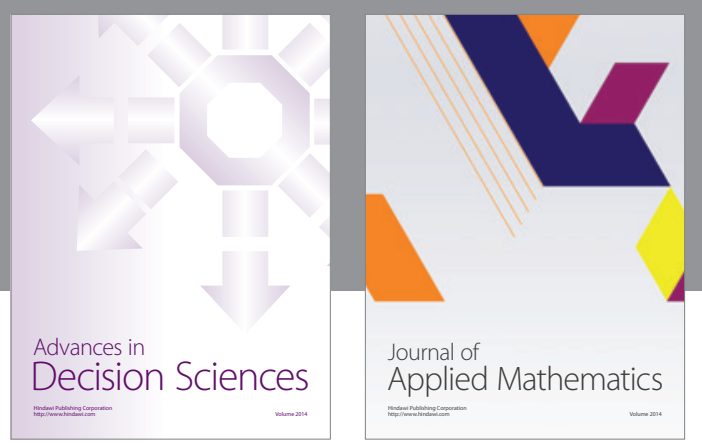

Algebra

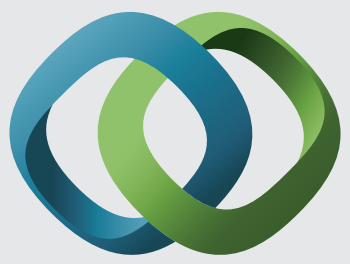

\section{Hindawi}

Submit your manuscripts at

http://www.hindawi.com
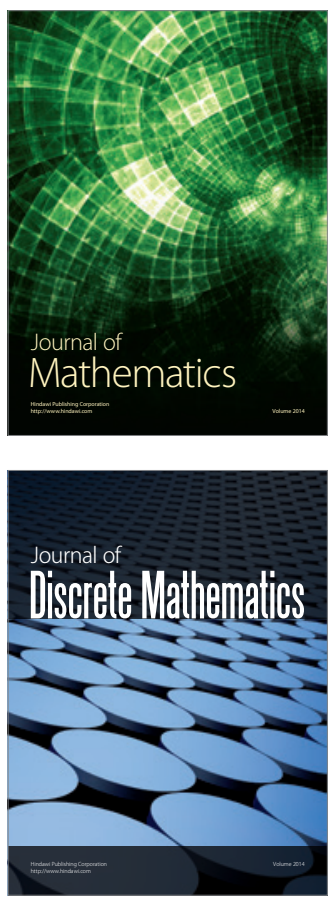

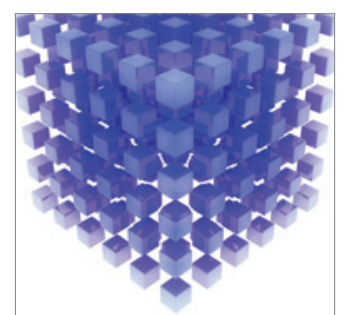

Mathematical Problems in Engineering
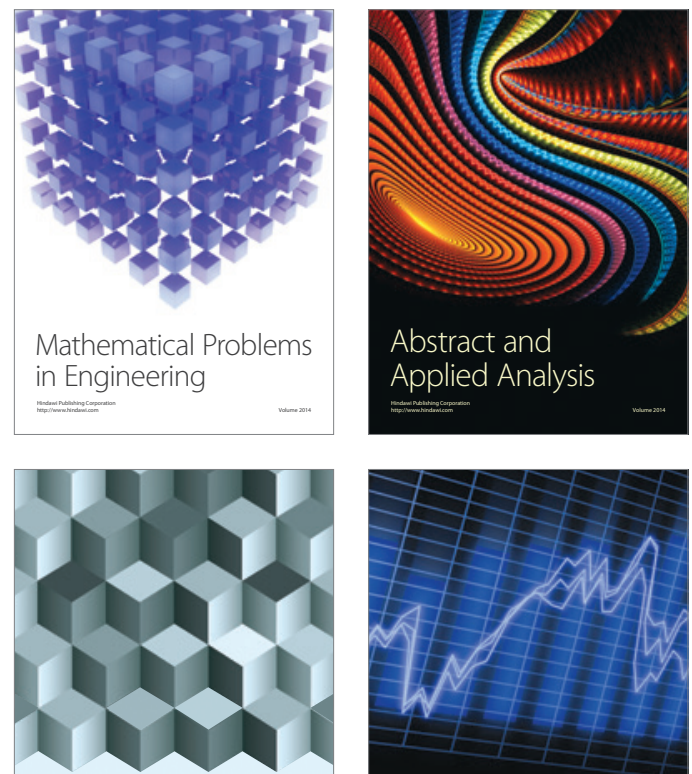

Journal of

Function Spaces

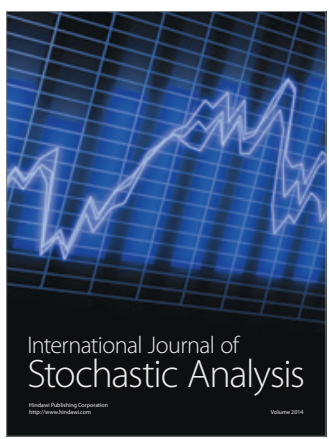

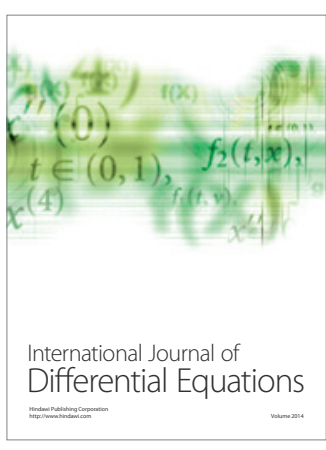
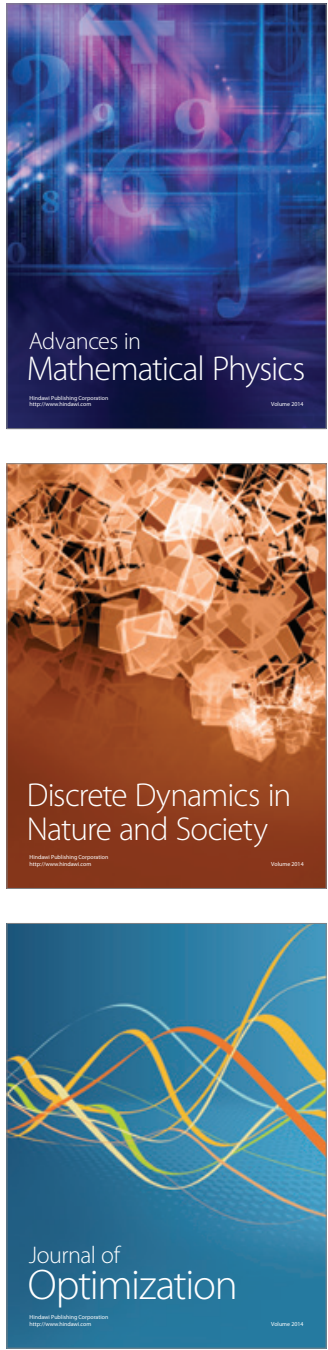